\title{
Fractal Model for Acoustic Absorbing of Porous Fibrous Metal Materials
}

\author{
Weihua Chen, ${ }^{1,2}$ Tianning Chen, ${ }^{1}$ Xiaopeng Wang, ${ }^{1}$ Jiuhui Wu, ${ }^{1}$ and Suobin $\mathrm{Li}^{1}$ \\ ${ }^{1}$ School of Mechanical Engineering and State Key Laboratory for Strength and Vibration of Mechanical Structures, \\ Xian Jiaotong University, Xian, Shaanxi 710049, China \\ ${ }^{2}$ College of Mechano-Electronic Engineering, Lanzhou University of Technology, Lanzhou 730050, China
}

Correspondence should be addressed to Xiaopeng Wang; xpwang@mail.xjtu.edu.cn

Received 1 May 2016; Accepted 10 August 2016

Academic Editor: Yuri S. Karinski

Copyright (C) 2016 Weihua Chen et al. This is an open access article distributed under the Creative Commons Attribution License, which permits unrestricted use, distribution, and reproduction in any medium, provided the original work is properly cited.

\begin{abstract}
To investigate the changing rules between sound absorbing performance and geometrical parameters of porous fibrous metal materials (PFMMs), this paper presents a fractal acoustic model by incorporating the static flow resistivity based on Biot-Allard model. Static flow resistivity is essential for an accurate assessment of the acoustic performance of the PFMM. However, it is quite difficult to evaluate the static flow resistivity from the microstructure of the PFMM because of a large number of disordered pores. In order to overcome this difficulty, we firstly established a static flow resistivity formula for the PFMM based on fractal theory. Secondly, a fractal acoustic model was derived on the basis of the static flow resistivity formula. The sound absorption coefficients calculated by the presented acoustic model were validated by the values of Biot-Allard model and experimental data. Finally, the variation of the surface acoustic impedance, the complex wave number, and the sound absorption coefficient with the fractal dimensions were discussed. The research results can reveal the relationship between sound absorption and geometrical parameters and provide a basis for improving the sound absorption capability of the PFMMs.
\end{abstract}

\section{Introduction}

Over the last two decades, the propagation characteristics of acoustic wave in porous fibrous metal materials (PFMMs) have attracted considerable attention because of their potential engineering applications [1-3]. The PFMM is a new type of multifunctional structural material and has demonstrated various novel physical properties, particularly the sound absorption under extreme conditions such as high sound pressure level and high temperature. Due to their excellent sound absorption performance and distinctive heat resistance, lightness, and stiffness, PFMMs are widely used in aircraft engine liners and combustion chambers for the rocket engines $[4,5]$.

In 1956, Biot [6, 7] constructed the constitutive equations and fluctuation control equations of porous materials for analyzing their acoustic properties. Cox and D'Antonio [8] studied the static flow resistivity of porous absorbers and summarized several empirical and semiempirical formulas for the parallel and perpendicular fibers, random fibrous arrangement, polyester fibers, and so forth. These empirical formulas can be used to evaluate the static flow resistivity. Kawasima [9] and Tarnow [10, 11] established the microstructure acoustic model and studied the sound propagation and the static flow resistivity of fibrous materials. It was shown that the static flow resistivity is important to describe sound propagation and attenuation in fibrous materials. Although the viscous dissipation of sound wave in a porous material depends on the thermal exchanges due to the compression and rarefaction instances between the air and the porous walls, the static flow resistivity dominates in viscous losses. Allard and Champoux [12] also found that the flow resistance of porous fibrous materials is closely related to the porous structure, but the accuracy of calculation for the static flow resistivity depends on the extent of approximation of the actual microstructures. In order to study the static flow resistivity of fibrous materials, based on a great deal of experimental data, Delany and Bazley [13] built an empirical relationship between the characteristic impedance and airflow resistivity. Sebaa et al. [14] used the reflected acoustic 


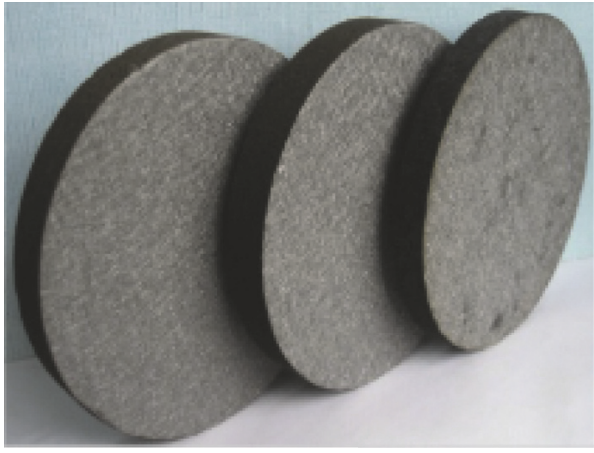

(a)

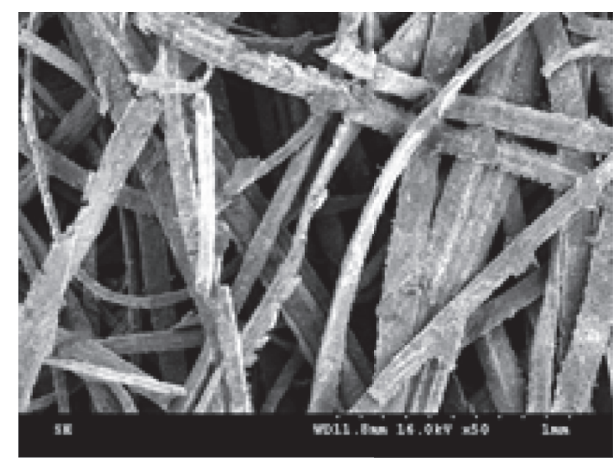

(b)

FIgURE 1: The PFMM samples: (a) photograph of the samples; (b) SEM photo of micrographic surface.

wave to measure the static flow resistivity of porous material and showed that the reflected wave is much more sensitive to the static flow resistivity than to porosity. Fellah et al. [15] introduced an acoustical transmission method to measure permeability of porous materials at low-frequency range. On the basis of a temporal model of the direct and inverse scattering problem, the proposed method is simple, rapid, and efficient. Experimental and numerical validation results of this method were presented. Sadouki et al. [16] established an acoustical method to measure static viscous permeability of porous absorbing materials. This method was derived without a priori estimation of the porosity, but by simply using the transmitted waves. Van der Kelen [17] investigated the static flow resistivity of two porous materials (fibrous glass wool and melamine foam) and the measurement method and inverse estimation were employed to determine the anisotropic flow resistivity. The research results showed that the homogeneities of the density and the static flow resistivity for the two materials are different but seem to have no correlation with each other. However, the sound propagation pathways in the PFMM are tortuous because of the irregular porous structure and there have been no theoretical models properly demonstrating the variation rules of the static flow resistivity with porous structures and pathways. Therefore, it is essential to present a quantitative description of the disordered pores in the PFMMs for evaluating the sound absorption performance.

Wang et al. [18] studied the microstructure features of $\mathrm{FeCrAl}$ fiber materials and obtained the fractal dimensions by the scanning electron microscope (SEM) photographs. The research results showed that the porous structure of the PFMM demonstrated obvious fractal features. Figure 1(a) displays the photographs of the PFMM samples and their microstructures (fibrous clusters (at macrolevel) and fibers (at microlevel)) are shown in Figure 1(b).

Based on the foregoing discussion, it can be found that a quantitative description of the disordered PFMM structure is crucial to the evaluation of the static flow resistivity. Allard and Atalla [19] established the equivalent fluid model, considering the impacts of heat conduction, air viscosity, and the structure factor on sound propagation. However, these parameters are difficult to measure directly. The static flow resistivity is a key parameter which is a function of the complex porous structure and porosity. Based on the similarity of aperiodic characters between the PFMM and sandstone or islands on the earth, they are analogous for porous structure and the arrangement of pores $[20,21]$. Fractal techniques have been used in diverse engineering applications involving physical phenomenon in disordered structures and multiple scales [22,23]. Therefore, it is possible to obtain the static flow resistivity formula of the PFMM by a fractal analysis on the porous microstructures.

On the basis of Biot-Allard model and the proposed static flow resistivity formula, a fractal acoustic model was presented to investigate the acoustic performance of the PFMM. We focus our attention on the derivation of a fractal acoustic model for the PFMM. The sound propagation pathways and porous microstructure are characterized by two fractal dimensions: one describing the tortuosity of the capillary pathways and the other relating the size of the capillary flow pathways to their quantity in the PFMM [24]. The fractal flow resistivity formula yields an analytical expression for the static flow resistivity which is a function of the two fractal dimensions and porosity of the PFMM. Moreover, the relationship between sound absorption performance and geometrical parameters can be obtained by the fractal acoustic model. The organization of the paper is as follows. In Section 2, the derivation of the fractal acoustic model and the static flow resistivity formula are presented, and the calculation of the tortuosity fractal dimension, the pore area fractal dimension, the maximum pore diameter, and the fibrous cluster radius is carried out in Section 3. In Section 4, the sound absorption is validated by experimental data and the influences of the fractal dimensions on the specific surface acoustic impedance, the complex wave number, and the sound absorption coefficient are discussed. The conclusions are drawn in Section 5.

\section{Acoustic Models}

2.1. Introduction of Biot-Allard Model. For the Biot-Allard model, a layer of porous material is assumed to be a layer of 
equivalent fluid with the effective density $\rho$ and bulk modulus $K$. The effective density $\rho$ can be written as [19]

$$
\rho=\alpha_{\infty} \rho_{0}\left(1+\frac{\sigma \phi}{j \omega \rho_{0} \alpha_{\infty}} G_{c}(s)\right),
$$

where $G_{c}(s)=-(s \sqrt{-j} / 4)\left(J_{1}(s \sqrt{-j}) / J_{0}(s \sqrt{-j})\right) /[1-(2 / s \sqrt{-j})$ $\left.\left(J_{1}(s \sqrt{-j}) / J_{0}(s \sqrt{-j})\right)\right]$ and $s=c\left(8 \omega \rho_{0} \alpha_{\infty} / \sigma \phi\right)^{1 / 2} ; \alpha_{\infty}$ is the tortuosity of the PFMM; $\rho_{0}$ is the static density of air; $\sigma$ is the static flow resistivity; $\phi$ is the porosity; $\omega$ is the angular frequency; and $c$ is the shape factor. In (1), the value of static flow resistivity is usually measured but the relationship between the static flow resistivity and geometrical parameters of the PFMM cannot be revealed.

In addition, the effective bulk modulus $K$ can be expressed as [19]

$$
K=\frac{\gamma P_{0}}{\gamma-(\gamma-1) F\left(B^{2} \omega\right)},
$$

where $F\left(B^{2} \omega\right)=\left[1+\left(\sigma \phi / j \omega B^{2} \rho_{0} \alpha_{\infty}\right) G_{c}(B s)\right]^{-1} ; B^{2}$ is Prandtl number; $\gamma$ is the air specific heat ratio; and $P_{0}$ is the static pressure of air. For air at $18^{\circ} \mathrm{C}, P_{0}=1.0132 \times 10^{5} \mathrm{~Pa} ; \rho_{0}=$ $1.213 \mathrm{~kg} \mathrm{~m}^{-3} ; \gamma=1.4$; and $B^{2}=0.71$.

Moreover, the surface acoustic impedance $Z_{s}$, the characteristic impedance $Z_{\mathrm{c}}$, and the complex wave number $k$ can be calculated, respectively, as follows [19]:

$$
\begin{aligned}
Z_{\mathrm{c}} & =\sqrt{K \rho}, \\
k & =i \omega \sqrt{\frac{\rho}{K}}, \\
Z_{\mathrm{s}} & =Z_{\mathrm{c}} \operatorname{coth}(k h),
\end{aligned}
$$

where $h$ is the thickness of the PFMM sample. Eventually, the sound absorption coefficient $\alpha$ at normal incidence is obtained as

$$
\alpha=1-\left|\frac{Z_{\mathrm{s}}-\rho_{0} c_{0}}{Z_{\mathrm{s}}+\rho_{0} c_{0}}\right|^{2} .
$$

In Biot-Allard model, the change rule between the effective density $\rho$ and the static flow resistivity $\sigma$ is revealed by (1). The static flow resistivity is used to predict the propagation constant and characteristic impedance of porous media through the empirical formulas. In this paper, a fractal acoustic model was presented based on the static flow resistivity with the tortuosity fractal dimension, the pore area fractal dimension, and the porosity of the PFMM.

\subsection{The Fractal Acoustic Model}

2.2.1. Fractal Geometry Theory. In general, regular objects such as point, curve, surface, and cube are described by integer dimensions 0, 1, 2, and 3 in Euclidean geometry. Each dimension is a measurement of an object such as the length of a line, the area of a surface, and the volume of a cube. However, a number of objects such as rough surface, coastline, mountain, river, and island on the earth are disordered and irregular, and they do not follow the Euclidean description due to the scale-dependent measurements of length, area, and volume [21]. These objects are called fractals and can be described using a nonintegral dimension called fractal dimension. The measurable quantity of a fractal object $M(\varepsilon)$ is related to the length scale $\varepsilon$ by the form [21]

$$
M(\varepsilon) \propto \varepsilon^{D}
$$

where $M(\varepsilon)$ means the length of a line, the area of a surface, the volume of a cube, or the weight of an object; $D$ is the fractal dimension. Equation (5) demonstrates the rule of self-similarity, which means the value of $D$ remains a constant over a range of length scales $\varepsilon$. Many geometric structures such as Koch curve and Sierpinski gasket are the examples of exact self-similar fractals. However, exact selfsimilarity objects in nature are seldom observed and the fractal description of many general objects is obtained based on statistical self-similarity [23]. The fractal dimension $D$ used in this paper refers to the statistical fractal dimension, which is usually estimated briefly as the slope of linear fit of data on a log-log plot of average measurement $M(\varepsilon)$ against the length scale $\varepsilon$. The concept of local, statistical similarity introduced above is used in the following section to demonstrate a geometric description of porous structure for the PFMM.

2.2.2. Fractal Characteristics of the PFMM. The porous structure of the PFMM is related to the fiber diameter and length and the arrangement of fibers. In reality, the fiber shape and agglomeration in the PFMM are irregular and disordered. As shown in Figure 2, $L_{0}$-sized cylindrical PFMM with various pore sizes can be regarded as a bundle of tortuous capillary tubes having different cross-sectional areas. The diameter of a capillary and its length along the incident sound wave are defined as $d$ and $L_{\mathrm{t}}(d)$, respectively, shown in Figure 2(a). Due to the tortuous nature of the capillary, $L_{\mathrm{t}}(d)$ is greater than or equal to $L_{0}$. The tortuosity of capillary depends on its diameter $d$; the smaller the capillary diameter, the greater its tortuous length, as illustrated schematically in Figures 2(a) and $2(\mathrm{~b})$.

The scaling relationship of the length $L$ and the tortuosity fractal dimension $D_{\mathrm{t}}$ by using the formula $L_{\mathrm{t}}(\varepsilon)=\varepsilon^{1-D_{\mathrm{t}}} L_{0}^{D_{\mathrm{t}}}$ was given by Wheatcraft and Tyler [25], where $\varepsilon$ is the length scale of measurement. By analogy with this model, the relationship between the diameter and the length of capillaries can be used to exhibit similar fractal scaling law as

$$
L_{\mathrm{t}}(d)=d^{1-D_{\mathrm{t}}} L_{0}^{D_{\mathrm{t}}}
$$

where $L_{\mathrm{t}}(d)$ is the total length of the tortuous capillary tube and $D_{t}$ is the tortuosity fractal dimension of the capillary path, which lies in the range $1<D_{t}<2$. A higher value of $D_{\mathrm{t}}$ corresponds to a highly tortuous capillary. Note that the capillary pathway is a straight path when the tortuosity fractal dimension $D_{\mathrm{t}}=1$. It corresponds to a highly tortuous line that sprawls a plane if the tortuosity fractal dimension $D_{\mathrm{t}}=2$. 


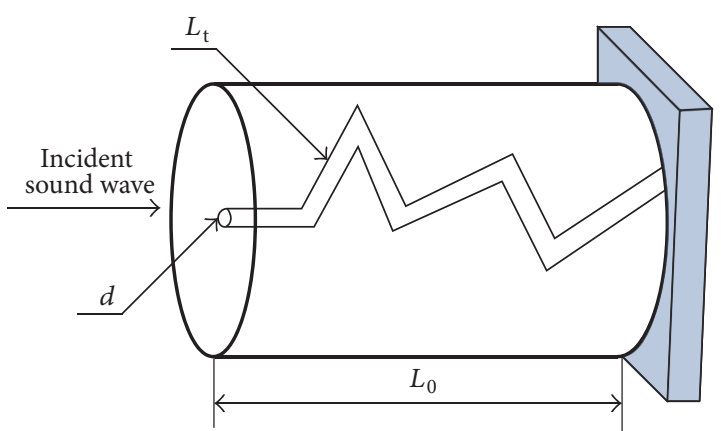

(a)

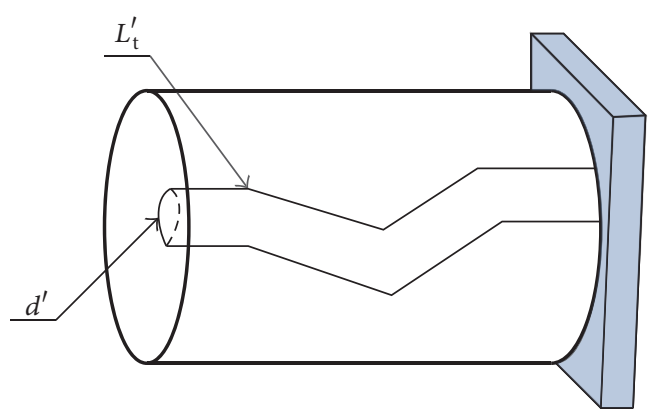

(b)

FIGURE 2: Schematic of sound propagation pathways of the PFMM. (a) The $d$-sized capillaries; (b) the illustration of the decrease in tortuosity with increasing capillary diameter.

The tortuosity of the capillary pathways can be described by (6), which reflects the fractal properties of porous structure.

On the other hand, in order to describe the porous structure of the PFMM, it is also important to calculate the number of capillary pathways with the pore diameter $d$ by considering a cross section of the PFMM perpendicular to the flow direction. According to the fractal theory, the total number of the islands whose area $A$ is greater than $a$ can be expressed by $N(A>a) \sim a^{-D / 2}$, where $D$ is the fractal dimension [21]. Assuming that the pores in the PFMM are analogous to the islands on the earth, the cumulative $d$-size pores also follow the same fractal scaling law [24]

$$
N(L \geq d)=\left(\frac{d_{\max }}{d}\right)^{D_{\mathrm{f}}}
$$

where $D_{\mathrm{f}}$ is the pore area fractal dimension and $d_{\max }$ is the maximum diameter of pores.

2.2.3. The Static Flow Resistivity Formula. As shown in Figure 1(b), the PFMM is composed of disordered metallic fibers connected with the sintered points. The fibrous materials may be regarded as transverse isotropic. Furthermore, because the density and stiffness of the fibers are much larger than those of the air in the porous materials, the propagation process for the sound in the PFMM can be treated as motionless. We assume that the sound wave remains in a plane when it is incident normally on the PFMM. In this paper, a static process for the sound propagation is applied.

As shown in Figure 3, the porous material is placed in a pipe and a pressure difference across the porous material induces a steady flow of air. The porous material is regarded as an infinite periodic structure and sound wave propagates unidirectionally from left to right. Based on the assumption of the static process, the airflow velocity in the pores is small so the nonlinear effects are not taken into account. The static flow resistivity of a homogeneous isotropic porous material is given by [19]

$$
\sigma=\frac{p_{2}-p_{1}}{V h}
$$

where $p_{2}$ is the incident pressure; $p_{1}$ is the exit pressure; $p_{2}-p_{1}$ is a pressure difference across the porous material;

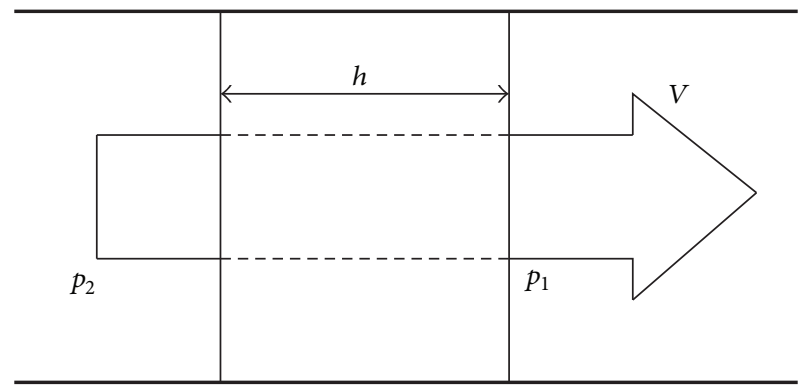

Figure 3: A differential pressure $p_{2}-p_{1}$ induces steady airflow $V$ of air per unit area of material.

$V$ is the mean flow velocity of air per unit area. The sound propagation through the PFMM mainly depends upon the static flow resistivity, the incident sound pressure difference, and the air viscosity.

In this section, we aim to obtain an analytical formula relating the static flow resistivity to the geometrical parameters because the static flow resistivity can be quantified by the tortuosity fractal dimension $D_{\mathrm{t}}$, the pore area fractal dimension $D_{\mathrm{f}}$, and the porosity $\phi$. For a unit cell which is composed of tubes with various cross-sectional areas, the total volumetric airflow rate $Q$ can be obtained by integrating the individual flow rate $q(d)$ over the whole pores. The flow rate $q(d)$, through a single tortuous capillary, is obtained by modifying the well-known Hagen-Poiseuille equation [26]

$$
q(d)=g_{\mathrm{q}} \frac{\Delta P}{L_{\mathrm{t}}(d)} \frac{d^{4}}{\mu},
$$

where $\mu$ is the viscosity of air and $g_{\mathrm{q}}$ is a geometric factor based on the shape of the capillary cross section. Further, the total flow rate $Q$ through the cross section of the PFMM is obtained by [27]

$$
Q=g_{\mathrm{q}} \frac{\Delta P}{\mu L_{0}^{D_{\mathrm{t}}}} \frac{D_{\mathrm{f}}}{3+D_{\mathrm{t}}-D_{\mathrm{f}}} d_{\max }^{3+D_{\mathrm{t}}}
$$


TABLE 1: The physical parameters of air used in this paper.

\begin{tabular}{lcccc}
\hline Air properties & $T_{0}(\mathrm{~K})$ & $\rho_{0}\left(\mathrm{~kg} \mathrm{~m}^{-3}\right)$ & $P_{0}(\mathrm{~Pa})$ & $\mu(\mathrm{Pa} \cdot \mathrm{s})$ \\
\hline Numerical values & 293 & 1.213 & $1.013 \times 10^{5}$ & $1.8 \times 10^{-5}$ \\
\hline
\end{tabular}

In addition, the total flow rate $Q$ passing through the PFMM can be calculated by Darcy's law

$$
\mathrm{Q}=A V,
$$

where $A$ is the total area of the cross section of the PFMM. Compared with the static flow resistivity formula, $V$ is also the mean flow velocity of air per unit area of material. The thickness of the PFMM is set as $L_{0}$. Substituting (10) and (11) into (8), the static flow resistivity formula can be obtained as

$$
\sigma=\frac{\mu A L_{0}^{D_{\mathrm{t}}-1}\left(3+D_{\mathrm{t}}-D_{\mathrm{f}}\right)}{g_{\mathrm{q}} D_{\mathrm{f}} d_{\max }^{3+D_{\mathrm{t}}}} .
$$

This demonstrates that the static flow resistivity $\sigma$ is a function of the tortuous fractal dimension $D_{\mathrm{t}}$, the pore area fractal dimension $D_{\mathrm{f}}$, and the geometrical parameters $A, L_{0}$, and $d_{\max }$. In addition, aiming to describe the convolute extent of sound propagating pathway, the concept of tortuosity is presented. As shown in Figure 4, the tortuosity of the sintered fibrous metal material is defined as [19]

$$
\alpha_{\infty}=\frac{1}{\cos ^{2} \varphi}=\left(\frac{L_{\mathrm{t}}(d)}{L_{0}}\right)^{2},
$$

where $\varphi$ is the angle between the axis of the pores and the surface. And then (6) can be modified as

$$
\frac{L_{\mathrm{t}}(d)}{L_{0}}=\left(\frac{L_{0}}{d}\right)^{D_{\mathrm{t}}-1}
$$

Substituting (14) into (13), the tortuosity formula related to the tortuosity fractal dimension can be deduced:

$$
\alpha_{\infty}=\left(\frac{L_{0}}{d}\right)^{2\left(D_{\mathrm{t}}-1\right)}
$$

Then, substituting (15) into (1) and setting $L_{0} / d=T$, we can get the effective density formula as

$$
\rho=\rho_{0} T^{2\left(D_{\mathrm{t}}-1\right)}\left(1+\frac{\sigma \phi}{j \omega \rho_{0} T^{2\left(D_{\mathrm{t}}-1\right)}} G_{c}(s)\right) .
$$

Therefore, the sound absorption performance of the PFMM can be calculated by using (2), (3), (4), (12), and (16). The physical parameters of air for the acoustic calculation in this paper are shown in Table 1.

\section{Determination of $d_{\max }, D_{\mathrm{t}}, D_{\mathrm{f}}$, and $R$}

3.1. Evaluation of $d_{\max }$. The architecture of the PFMM can be thought to be built with cylindrical fibers and fibrous clusters. The small pores are distributed between fibers and

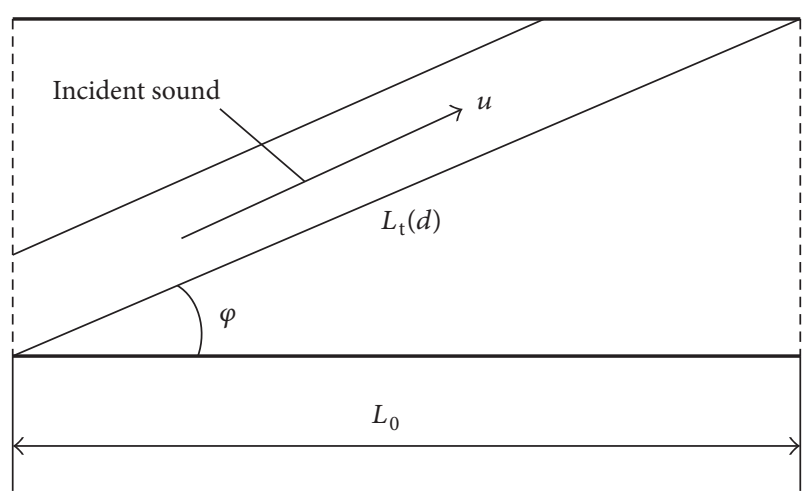

FIgURE 4: Schematic illustration of the PFMM with pores of constant and equal radius, with respect to the normal of surface.

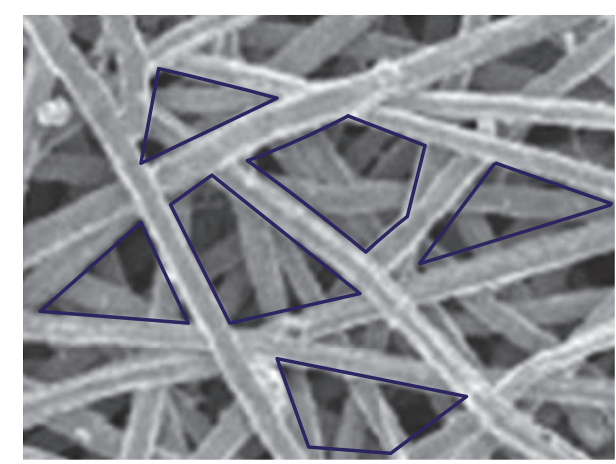

FIGURE 5: Schematic of the irregular porous shapes in the PFMM sample.

the large ones are formed between fibrous clusters. It can be seen from Figure 5 that the porous structures demonstrate different shapes, that is, triangular, quadrilateral, pentagonal, and so on. The calculation of the effective density $\rho$ was performed for cylindrical tubes having different cross sections corresponding to different shape factors [19]. The shape factor of equilateral triangle is set as 1.11. For the presented PFMM sample, the unit cell is assumed to be an equilateral triangle and its porosity is analyzed.

Due to the structural similarity between the PFMM and bidispersed porous media, the maximum pore diameter $d_{\max }$ can be approximately given by [27]

$$
d_{\max }=R \sqrt{2\left(\frac{1-\phi_{i}}{1-\phi}-1\right)},
$$

where $R$ is the radius of fibrous clusters; $\phi_{i}$ is the porosity of fibrous cluster; and $\phi$ is the porosity of PFMM sample.

\subsection{Determination of $D_{\mathrm{t}}$ and $D_{\mathrm{f}}$}

3.2.1. Evaluation of $D_{\mathrm{t}}$. In this section, we present two methods for the calculation of $D_{\mathrm{t}}$ : one is the determination by employing the fractal relationship between the total distance the sound wave travels and the straight-line distance along the $x$-axis and the other one is the box-counting method. Figure 6 


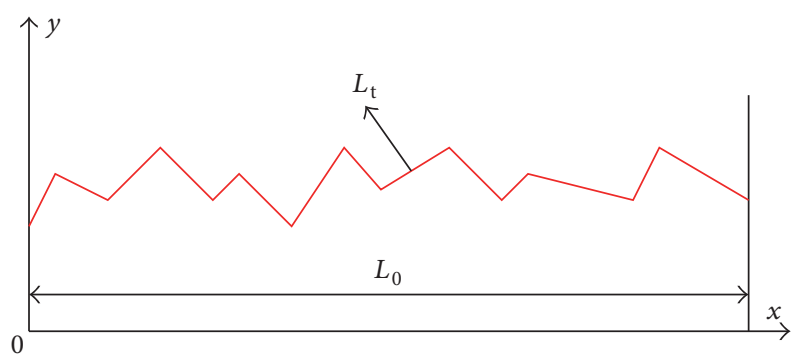

FIGURE 6: The illustration of sound propagation pathway $L_{\mathrm{t}}$ versus the thickness $L_{0}$ of the sample.

plots an $x-y$ coordinate system on the fractal pathway and the sound wave propagates along the $x$-axis. According to Monte Carlo simulations, Wheatcraft and Tyler [25] presented an average fractal travel distance $L_{\mathrm{t}}(\lambda)$ versus the scale $\lambda$ by the fractal random walk model to simulate the dispersity in heterogeneous media. They obtained the tortuosity fractal dimension $D_{\mathrm{t}}=1.081$.

For the present analysis, it is assumed that the fractal dimension is a constant when the value of $x$ is assigned from 0 to $L_{0}$. The relationship between $L_{\mathrm{t}}$ and $L_{0}$ can be obtained by (6). By taking natural logarithms on both sides of (6), the $D_{t}$ formula can be obtained as

$$
D_{\mathrm{t}}=\frac{\ln L_{\mathrm{t}}-\ln d}{\ln \left(L_{0} / d\right)} .
$$

To evaluate the tortuosity fractal dimension $D_{t}, L_{0}$ is set as the thickness of sample; $L_{\mathrm{t}}$ and $d$ are measured by the microstructure of the PFMM sample. For instance, the approximate measured parameters $L_{\mathrm{t}}=32 \mathrm{~mm}, d=0.5 \mathrm{~mm}$, and $L_{0}=25 \mathrm{~mm}$ can be used for our considered samples. The calculated value of the tortuosity fractal dimension $D_{t}$ is 1.063 .

Next, the tortuosity fractal dimension is determined by the box-counting method. We can approximately simulate the sound propagation pathways by employing the fractal random walk model. Figure 7 shows five random pathways for the sound propagation in the PFMM when the sound wave is incident from left to right.

The fractal software FractalFox was used to calculate the tortuosity fractal dimension of the sound propagation pathways shown in Figure 7(a). By this method, the cross section is discretized by square boxes of size $L$, and the number of such $L$-sized boxes along the perimeter of the pore regions is recorded as $N(L)$. This step is repeated for various box sizes, and a ln-ln plot of $N(L)$ versus $L$ is constructed. The slope of the best-fit straight line indicates the tortuosity fractal dimension. As for the first sound propagation pathway in Figure 7(a), the calculated procedure of the tortuosity fractal dimension is as follows: (1) transform the format of the graph for the pathway to JPG format and import the new photo to the FractalFox software; (2) set the initial box size and the final box size as $5 \mathrm{~mm}$ and $50 \mathrm{~mm}$, respectively, and the step size is set as $5 \mathrm{~mm}$; (3) select the box-counting method and run the "L. Current Fractal" order. Then, the regression data of the number of boxes and box sizes can be obtained. Finally, the tortuosity fractal dimension is obtained by the slope of the best-fit straight line of the $\ln$-ln plot of $N(L)$ versus $L$.

Figure 7(b) illustrates the calculated result of the tortuosity fractal dimension for the first pathway and $D_{t}=1.05$. By repeating the same procedure, the calculated results of the tortuosity fractal dimensions for other sound propagation pathways are $1.08,1.02,1.07$, and 1.10 , respectively. The average value $D_{\mathrm{t}}=1.06$ is very close to the theoretical calculated value.

3.2.2. Calculation of $D_{\mathrm{f}}$. The pore area fractal dimension can be obtained through an analysis of microstructural images of the PFMM's cross sections [28]. The SEM photographs of the PFMM for the fractal analysis are shown in Figure 8. The box-counting method is used to calculate the pore area fractal dimension and the calculation procedure for the FractalFox software is presented. The number of boxes $N(L)$ is required to cover the pore area completely.

Similarly, the pore area fractal dimension is also determined by the slope of linear fit through the data on a $\ln -\ln$ plot of $N(L)$ versus $L$. The logarithmic plots of the cumulative number of pores $N(L \geq d)$ versus $d$ are shown in Figure 9 $(\phi=0.81,0.86$, and 0.90$)$. It can be seen that the number of the cumulative pores decreases with the pore size.

As shown in Figure 9, the pore area fractal dimension can be obtained as $D_{\mathrm{f}}=1.49$ (Figure 9(a)), $D_{\mathrm{f}}=1.57$ (Figure $9(\mathrm{~b})$ ), and $D_{\mathrm{f}}=1.59$ (Figure $9(\mathrm{c})$ ).

3.3. Fibrous Clusters Radius R. Based on the microstructure photographs of the PFMM samples, we measured the radius of fibrous clusters by SEM. As shown in Figure 10, we obtained the SEM photographs ( $\times 50$ times $)$ of the PFMM samples. Then, the particle size measurement software SmileView was employed to measure the radius of fibrous clusters. The procedure of the calculation of the radius of fibrous clusters is as follows: (1) import the SEM photograph to the software SmileView; (2) set the scale of the measurement as $1: 1000(\mu \mathrm{m}) ;(3)$ measure the radius of fibrous clusters.

Figure 11 shows the photographs of the micrographic surface and the radius of fibrous clusters for each sample is measured five times. As shown in Table 2, the measured values of average radii are 359,355 , and $346 \mu \mathrm{m}$.

\section{Sound Absorption Verification and Discussion}

In this section, the established fractal acoustic model is validated by the values of Biot-Allard model and experimental data. We measured the values of the static flow resistivity for three PFMM samples and the static flow resistivity formula is validated.

4.1. Experiment and Verification of $\sigma$. Two measuring methods of the static flow resistivity are introduced by the ISO 9053 standard [29]: one is steady airflow method and the other is alternating airflow method. In this section, we used the steady airflow method to measure the static flow resistivity. Figure 12(a) shows a simplified sketch of the device for the 


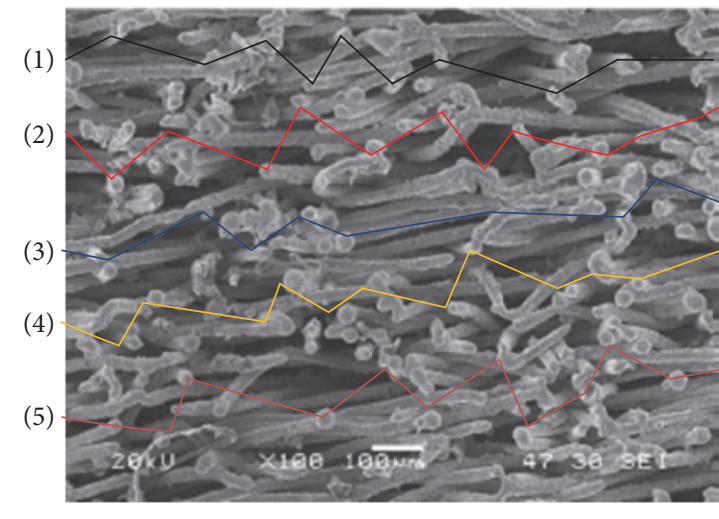

(a)

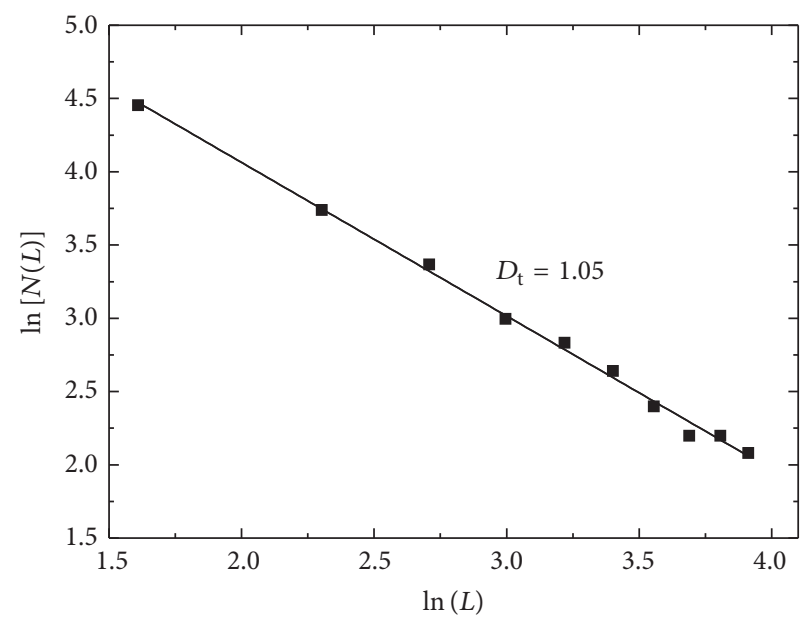

(b)

Figure 7: The results of the tortuosity fractal dimension $D_{\mathrm{t}}$ calculated by the box-counting method. (a) Five possible random sound propagation pathways; (b) the result of $D_{\mathrm{t}}$ for the first pathway.

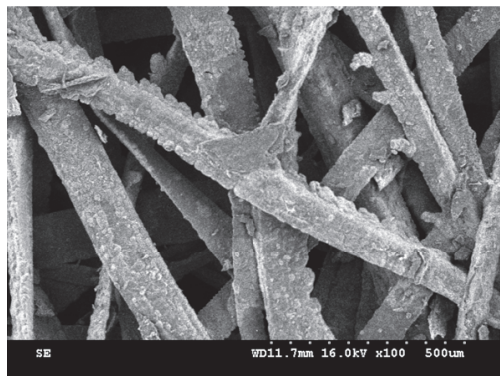

(a)

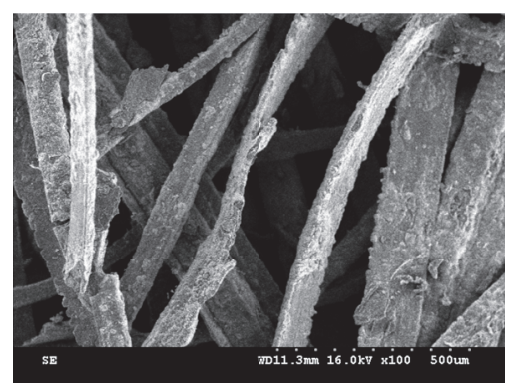

(b)

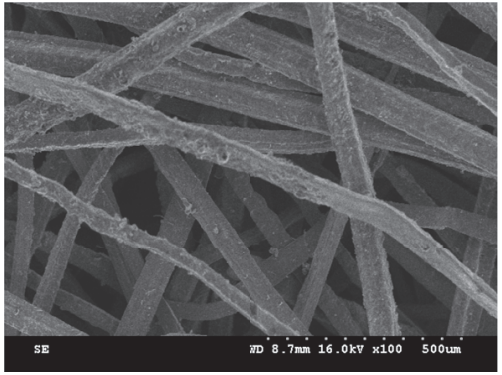

(c)

FIGURE 8: The SEM photographs of the PFMM’s cross sections: (a) sample \#1; (b) sample \#2; (c) sample \#3.

steady airflow resistivity measurement and Figure 12(b) is blanked since it is an in-house apparatus of the Institute of Acoustics at the Chinese Academy of Sciences and was published without their permission.

The setup is made up of differential gauge, flow meters, and specimen tube. The inner diameter of the measuring tube is greater than $95 \mathrm{~mm}$ and the minimum air velocity can reach $0.5 \times 10^{-3} \mathrm{~m} / \mathrm{s}$. The measured values of the static flow resistivity for three PFMM samples are shown in Table 3.

Furthermore, we calculated the static flow resistivity of the PFMM in terms of the presented static flow resistivity formula considering the influences of the tortuous fractal dimension, the pore area fractal dimension, and the porosity. Firstly, in order to evaluate the average effect of the tortuosity fractal dimension on the static flow resistivity, we calculated the values of the static flow resistivity and the deviations between calculated results and experimental data for three PFMM samples with $D_{\mathrm{t}}=1.02,1.05,1.07,1.08$, and 1.10, respectively. The calculated values and the deviations are shown in Table 4.

It can be seen that the maximum absolute deviations of the static flow resistivity (sample \#1 to sample \#3) are
$15.21 \%, 19.14 \%$, and $15.55 \%$, respectively; the minimum absolute deviations are $1.67 \%, 0.08 \%$, and $2.41 \%$, respectively. Particularly, the deviations of the static flow resistivity calculated by employing the average tortuosity fractal dimensions are $1.95 \%, 3.63 \%$, and $3.96 \%$, respectively. Therefore, the calculations for the static flow resistivity by using the average value of $D_{t}$ can reduce the stochastic errors and improve the prediction accuracy.

In addition, to evaluate the average effect of fibrous radius $R$, the static flow resistivities with different fibrous radii are calculated. The calculated values and the deviations of the static flow resistivity are shown in Table 5 . The results show that the maximum absolute deviations of the static flow resistivity are $17.85 \%, 17.23 \%$, and $22.32 \%$, respectively; the minimum absolute deviations are $0.83 \%, 6.75 \%$, and $4.77 \%$, respectively. The average errors are $1.95 \%, 1.93 \%$, and $2.41 \%$, respectively. It can be concluded that the calculations of the static flow resistivity by employing the average radii are reasonable.

Next, the tortuosity fractal dimension $D_{t}$ is set as 1.06 and the pore area fractal dimensions $D_{\mathrm{f}}$ are set as 1.49 , 1.57 , and 1.59, respectively. The shape factor $g_{\mathrm{q}}$ is set as 1.11 


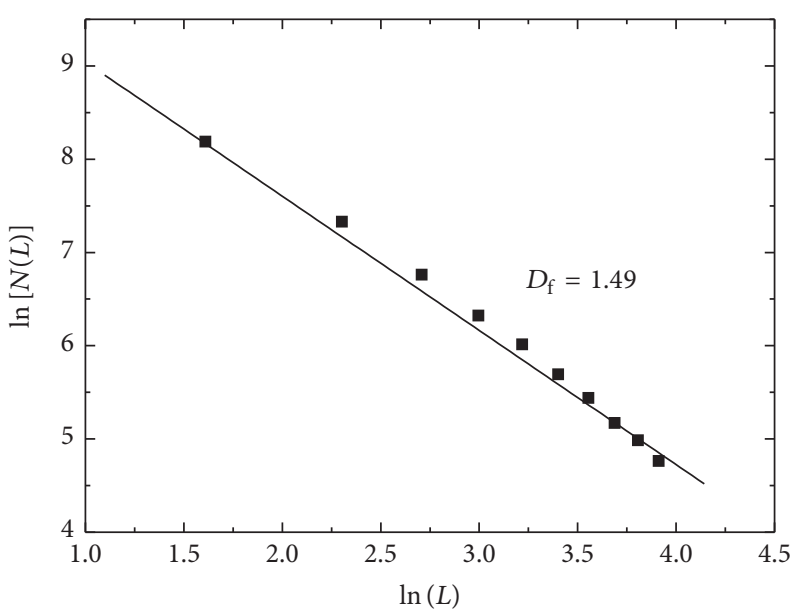

(a)

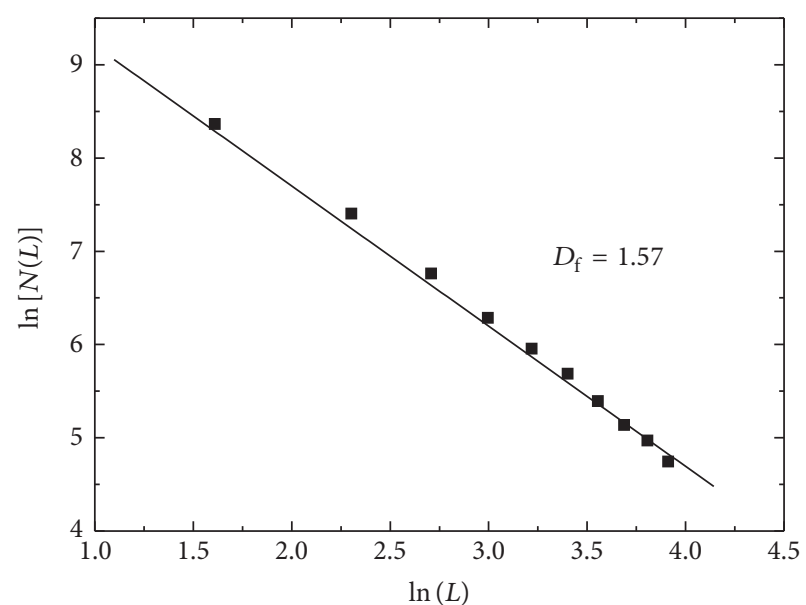

(b)

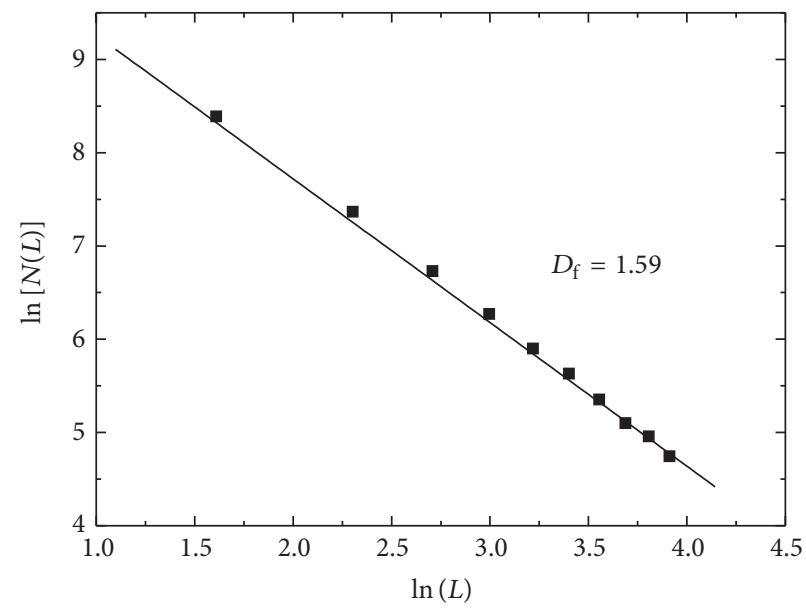

(c)

FIGURE 9: The calculated results of the pore area fractal dimension $D_{\mathrm{f}}$ for the PFMM samples.

TABLE 2: The measuring results of $R$ for fibrous clusters (unit: $\mu \mathrm{m}$ ).

\begin{tabular}{lcccccc}
\hline Fiber clusters & $R_{1}$ & $R_{2}$ & $R_{3}$ & $R_{4}$ & $R_{5}$ & Mean value \\
\hline Sample \#1 & 354 & 358 & 363 & 347 & 375 & 359 \\
Sample \#2 & 343 & 347 & 371 & 351 & 354 & 335 \\
Sample \#3 & 336 & 340 & 366 & 353 & 346 \\
\hline
\end{tabular}

and the fibrous cluster radii are $3.59 \times 10^{-4} \mathrm{~m}, 3.55 \times 10^{-4} \mathrm{~m}$, and $3.46 \times 10^{-4} \mathrm{~m}$, respectively. And then the calculated results of the static flow resistivity of three PFMM samples are compared with experimental data. Figure 13(a) shows the comparisons between the calculated results and experimental data at different porosities. It can be seen that the theoretical results agree well with the experimental data.

Due to the limitation of our experimental data, the static flow resistivity formula has been further verified by the calculated values in the literature. The physical parameters of the PFMM and the calculated data are shown in Table 6.

Figure 13(b) displays the comparisons between the results evaluated by the static flow resistivity formula and the data in
[30]. It can be shown that the static flow resistivity decreases with the porosity and the results calculated by the theoretical formula coincide very well with the literature's data, which proves the accuracy of the static flow resistivity formula.

4.2. Sound Absorption Verification. In order to validate the fractal acoustic model, we measured the sound absorption coefficients of three PFMM samples. The tested samples are made of stainless steel fibers with the same diameter and the thickness is $25 \mathrm{~mm}$. The sound absorption coefficients are measured by employing the transfer function method and the tested setup is B\&K 4206 impedance tube. 
TABLE 3: The physical parameters of the PFMM samples and experimental results of static flow resistivity.

\begin{tabular}{lccccc}
\hline Sample parameters & $\begin{array}{c}\text { Porosity } \\
\phi(\%)\end{array}$ & $\begin{array}{c}\text { Fiber diameter } \\
d(\mu \mathrm{m})\end{array}$ & $\begin{array}{c}\text { Thickness } \\
h(\mathrm{~mm})\end{array}$ & \multicolumn{2}{c}{$\begin{array}{c}\text { Shape factor } \\
g_{\mathrm{q}}\end{array}$} \\
\hline Sample \#1 & 81 & 50 & 25 & 1.11 & $\begin{array}{c}\text { Flow resistivity } \\
\sigma\left(\mathrm{Nm}^{-4} \mathrm{~s}\right)\end{array}$ \\
Sample \#2 & 86 & 50 & 25 & 1.11 & 98756 \\
Sample \#3 & 90 & 50 & 25 & 1.11 & 34655 \\
\hline
\end{tabular}

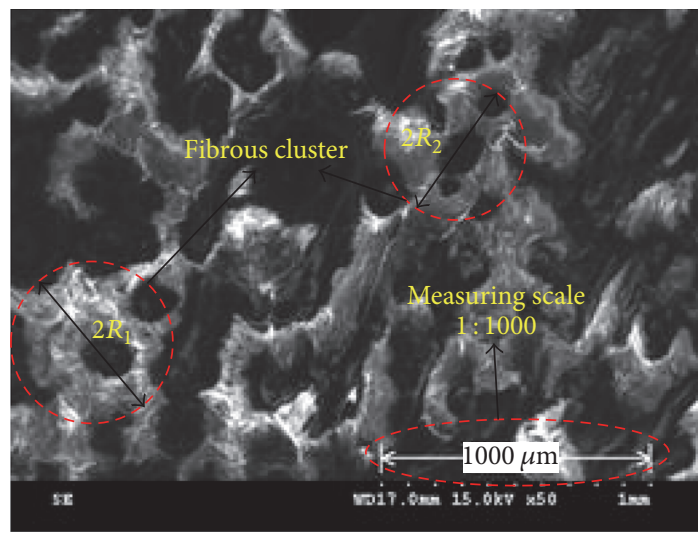

Figure 10: Typical porous morphology of fibrous clusters where $R$ denotes the fibrous cluster radius and the measuring scale of calculation is $1: 1000$.

Figure 14 plots the predictions evaluated by the fractal acoustic model and Biot-Allard model compared with experimental data. It can be seen that the theoretical results for sound absorption agree well with experimental data. As shown in Figure 14, the values calculated by the fractal acoustic model fit the measured ones better than BiotAllard model at low frequencies. The small deviations may be attributed to the computational errors of $D_{\mathrm{t}}$ and $D_{\mathrm{f}}$. In summary, the sound absorption coefficient of the PFMM increases with the frequency. In frequency domain, the sound absorption coefficient increases monotonically from 100 to $2000 \mathrm{~Hz}$ but decreases from 2000 to $4000 \mathrm{~Hz}$. When the frequency is higher than $4000 \mathrm{~Hz}$, the sound absorption coefficient is increased. For the sound absorption of the PFMM, the peak value of the curve is mainly formed by the resonance between sound wave and the PFMM. What is more, the PFMM has superior capability of energy dissipation for high-frequency sound wave and the sound absorption coefficient reaches a peak value when the energy dissipation ability of the PFMM approaches its maximum value.

4.3. Influences of Fractal Dimensions. In this section, the influences of the tortuosity fractal dimension and the pore area fractal dimension on the specific surface acoustic impedance $Z_{\mathrm{s}} / Z_{0}$, the complex wave number $k$, and the sound absorption coefficient $\alpha$ are presented and discussed.

4.3.1. Influences of $D_{\mathrm{t}}$ and $D_{\mathrm{f}}$ on $Z_{\mathrm{s}} / Z_{0}$. Figure 15 plots the real and imaginary parts of $Z_{s} / Z_{0}$ for sample \#1 with $D_{\mathrm{t}}=$ 1.06, 1.08, and 1.10, respectively. As shown in Figure 15(a), the specific acoustic resistance (the real part of $Z_{\mathrm{s}} / Z_{0}$ ) increases with the tortuosity fractal dimension in lowfrequency range but decreases in high-frequency range. Furthermore, the peak value of the real part of $Z_{\mathrm{s}} / Z_{0}$ curve is increased with the tortuosity fractal dimension and moves to the low frequency. Figure 15(b) shows that the specific acoustic reactance (the imaginary part of $Z_{\mathrm{s}} / Z_{0}$ ) decreases slightly with the tortuosity fractal dimension in low-frequency range but increases in high-frequency range. In other words, the specific acoustic resistance changes obviously with the increase of $D_{\mathrm{t}}$ but the specific acoustic reactance changes slightly in the whole frequency range.

On the other hand, the influences of the pore area fractal dimension on the specific surface acoustic impedance $Z_{\mathrm{s}} / Z_{0}$ are illustrated in Figure 16.

As shown in Figure 16(a), the specific acoustic resistance decreases with the pore area fractal dimension in lowfrequency range but increases in high-frequency range. The peak value of the real part of $Z_{\mathrm{s}} / Z_{0}$ curve increases with the pore area fractal dimension and moves to the high-frequency range. Figure 16(b) shows that the specific acoustic reactance decreases slightly with the pore area fractal dimension in lowfrequency range but increases in high-frequency range.

4.3.2. Influences of $D_{\mathrm{t}}$ and $D_{\mathrm{f}}$ on $k$. The changing rules between the complex wave number and the fractal dimensions are discussed in this section. Figure 17 shows that both the real and the imaginary parts of the complex wave number increase with the tortuosity fractal dimension. The real part of $k$ increases with the frequency but the imaginary part of $k$ decreases.

Figure 18 illustrates the variation of the complex wave number with the pore area fractal dimension. As shown in Figure 18(a), the real part of $k$ increases with the frequency and decreases with the pore area fractal dimension. Figure 18(b) shows that the imaginary part of $k$ decreases with the frequency and finally keeps constant. Furthermore, the imaginary part of $k$ decreases with the pore area fractal dimension.

4.3.3. Influences of $D_{\mathrm{t}}$ and $D_{\mathrm{f}}$ on $\alpha$. The effects of $D_{\mathrm{t}}$ and $D_{\mathrm{f}}$ on the sound absorption coefficient $\alpha$ are plotted in Figure 19. As shown in Figure 19(a), the variations of the sound absorption coefficient with different tortuosity fractal dimensions $\left(D_{\mathrm{t}}=\right.$ $1.06,1.08$, and 1.10) and $D_{\mathrm{f}}=1.49$ are plotted. The results show that the sound absorption coefficient increases with the tortuosity fractal dimension in $500-2500 \mathrm{~Hz}$ and $4800-$ $6400 \mathrm{~Hz}$ ranges but decreases in $2500-4800 \mathrm{~Hz}$ range. With the increasing of the tortuosity fractal dimension, the fluid 


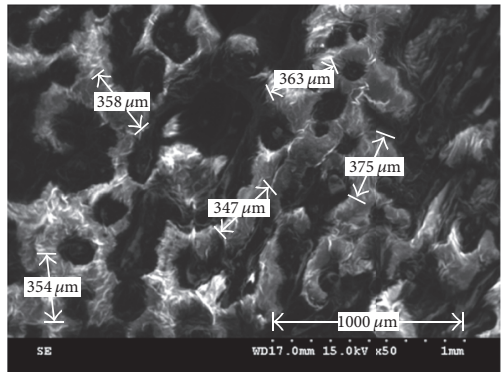

(a)

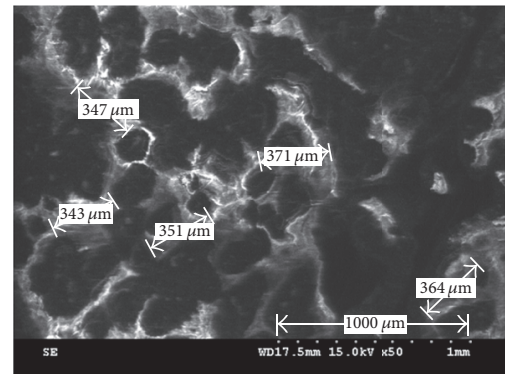

(b)

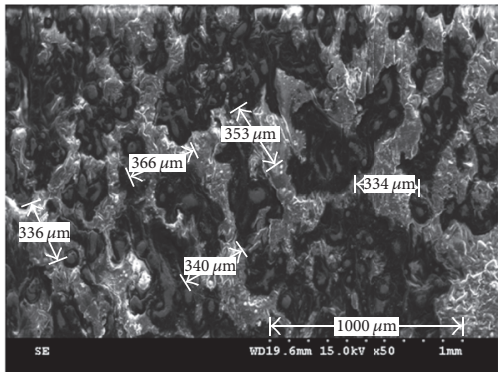

(c)

FIGURE 11: The photographs of the micrographic surface for $R$ measuring: (a) sample \#1; (b) sample \#2; (c) sample \#3.

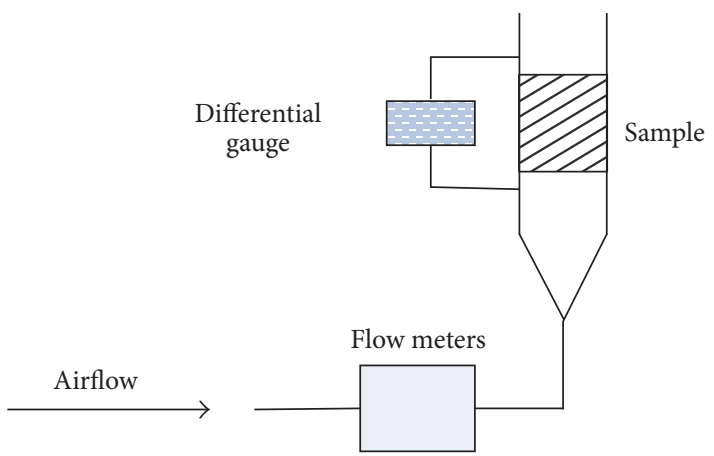

(a) (b)

Figure 12: The static flow resistivity measurement. (a) Schematic of the setup. (b) This Figure is blanked since it is an in-house apparatus of the Institute of Acoustics at the Chinese Academy of Sciences and was published without their permission.

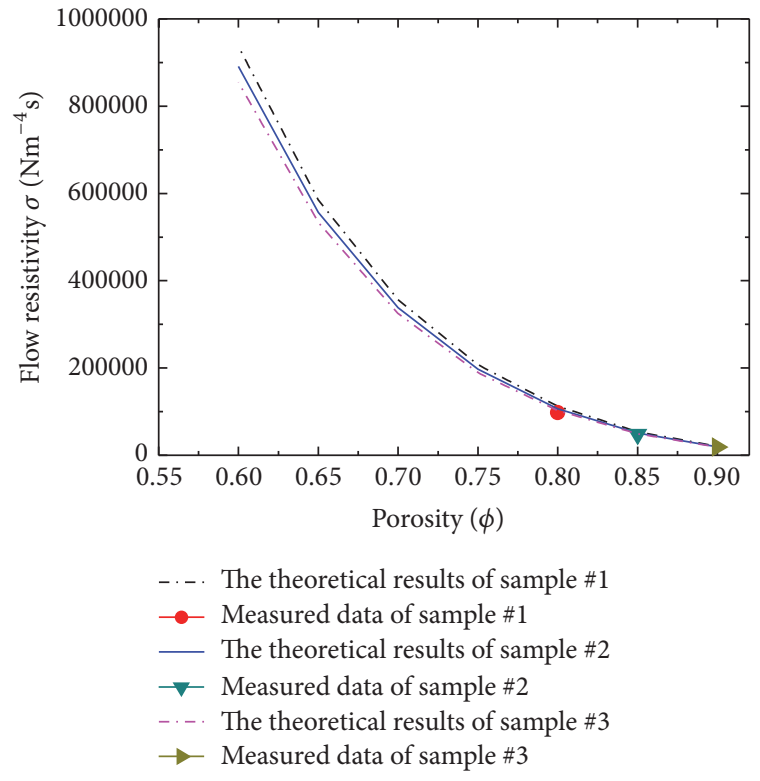

(a)

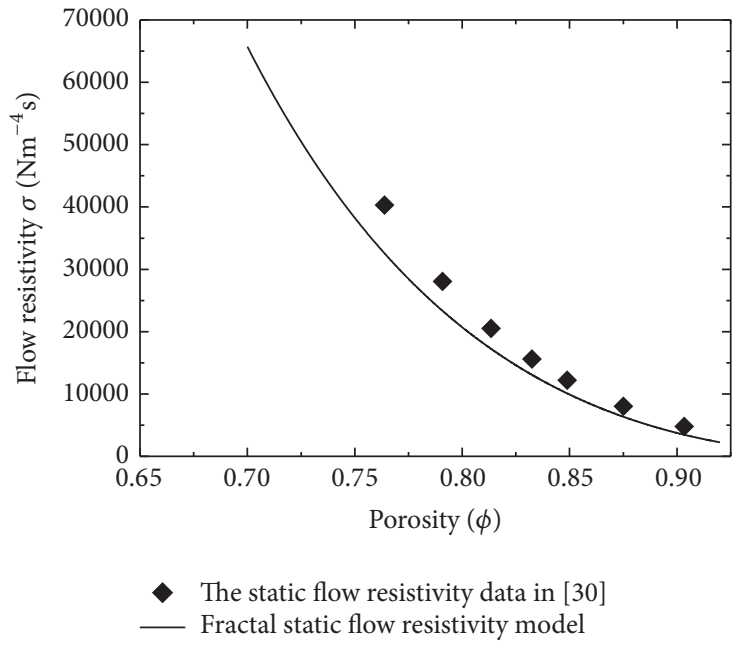

(b)

FIGURE 13: Comparisons between the calculated and experimental results of the static flow resistivity: (a) compared with the test data; (b) compared with the calculated results in [30]. 


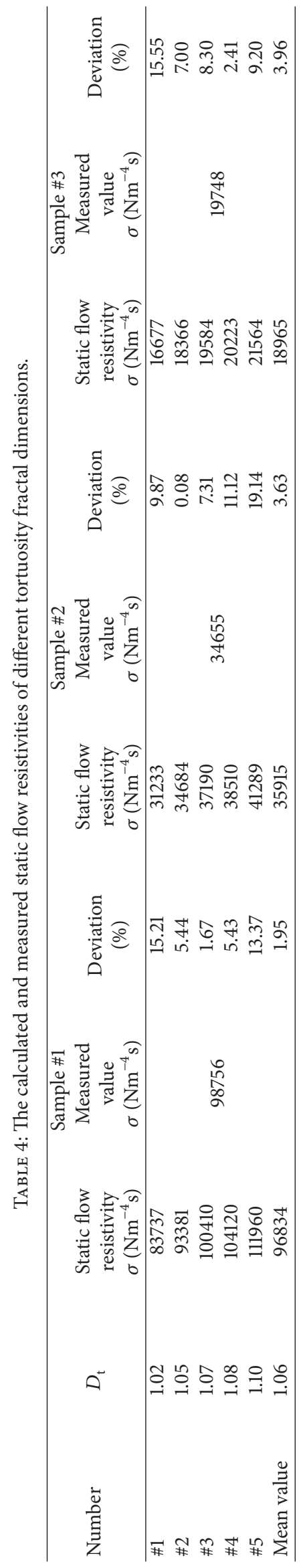




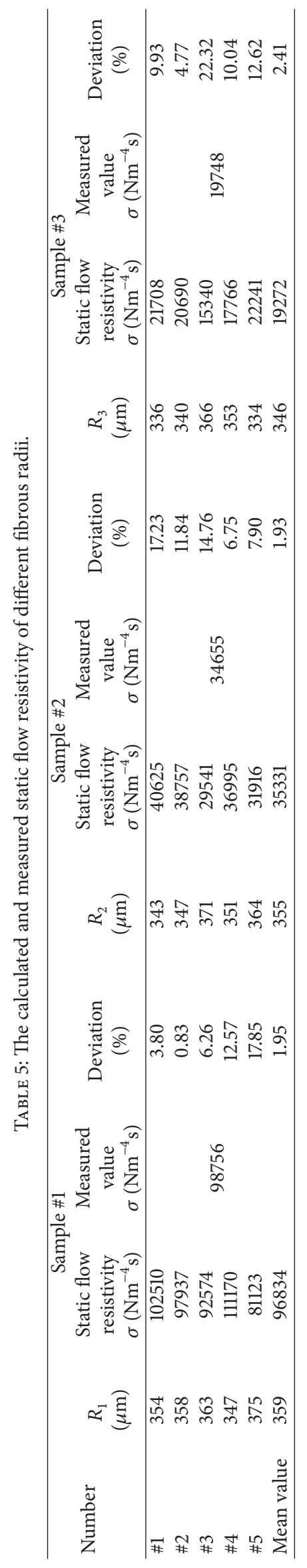



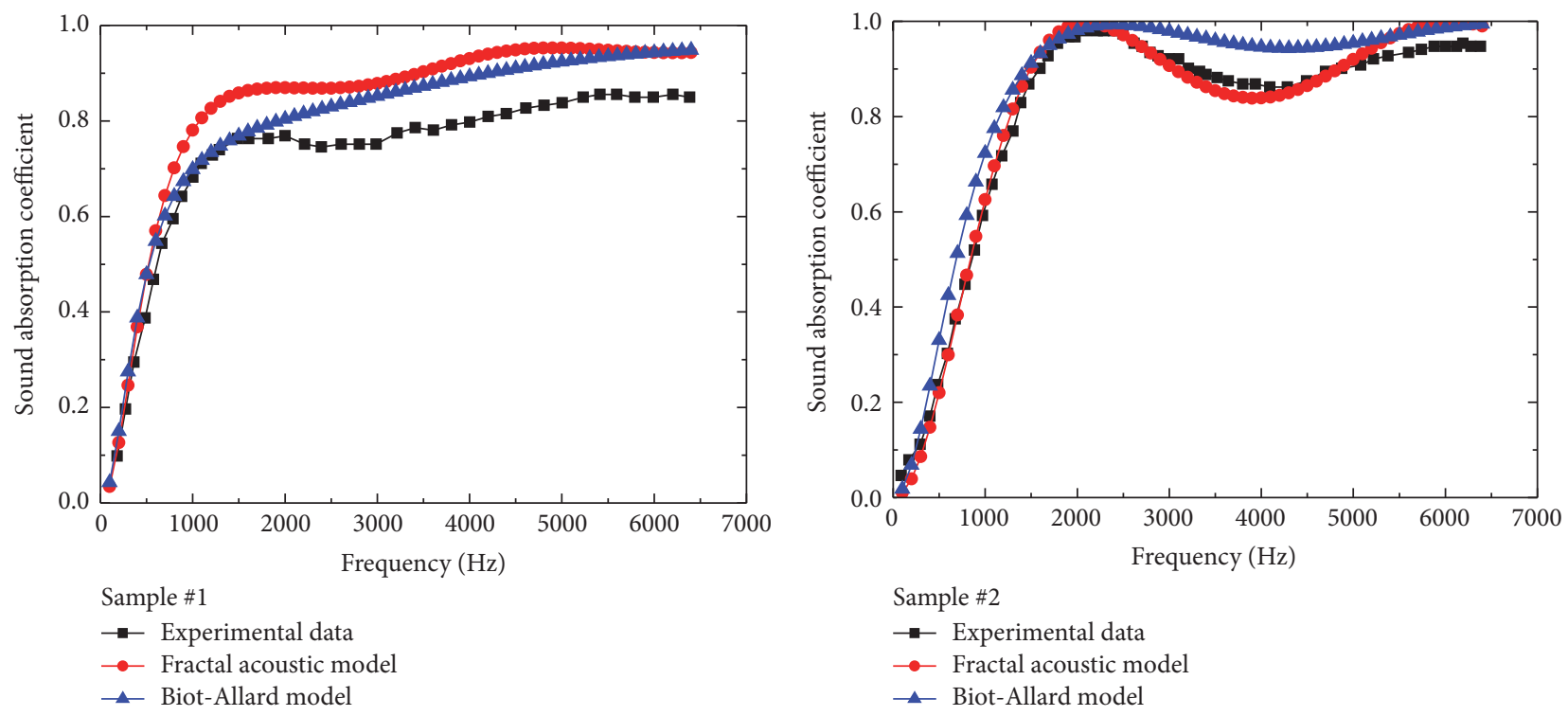

(a)

(b)

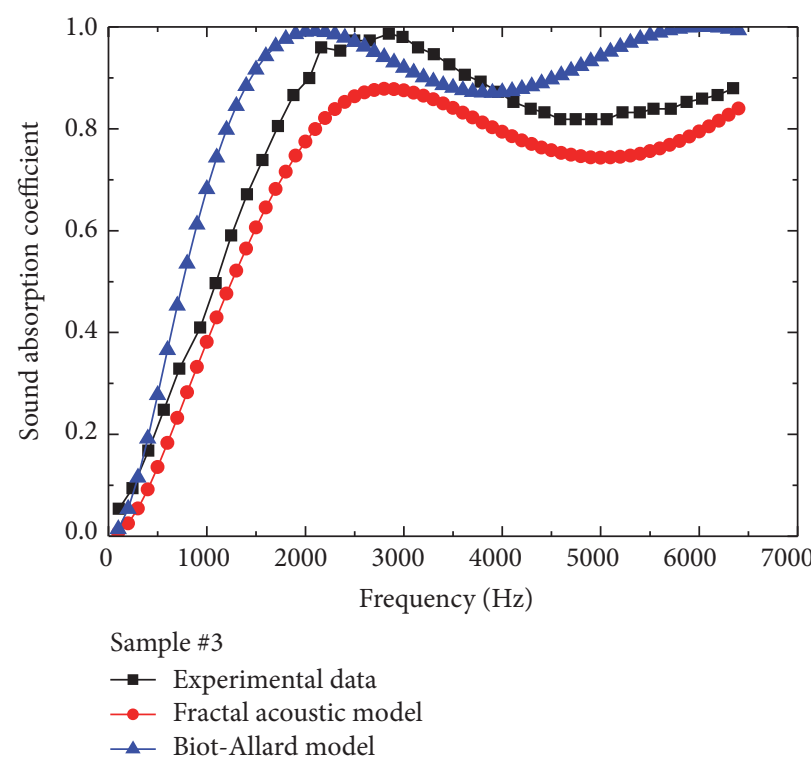

(c)

Figure 14: Comparisons between the calculated and measured sound absorption coefficients of the PFMM samples: (a) sample \#1, (b) sample $\# 2$, and (c) sample \#3.

TABLE 6: The physical parameters of the porous metallic samples and calculated static flow resistivities.

\begin{tabular}{lccccc}
\hline $\begin{array}{l}\text { Sample } \\
\text { number }\end{array}$ & $\begin{array}{c}\text { Porosity } \\
\phi(\%)\end{array}$ & $\begin{array}{c}\text { Fiber radius } \\
r(\mu \mathrm{m})\end{array}$ & $\begin{array}{c}\text { Thickness } \\
h(\mathrm{~mm})\end{array}$ & $\begin{array}{c}\text { Tortuosity } \\
\alpha_{\infty}\end{array}$ & $\begin{array}{c}\text { Static flow } \\
\text { resistivity } \\
\sigma\left(\mathrm{Nm}^{-4} \mathrm{~s}\right)\end{array}$ \\
\hline$\# 1$ & 76.38 & 50 & 25 & 1.12 & 40323 \\
$\# 2$ & 79.08 & 50 & 25 & 1.11 & 28057 \\
$\# 3$ & 81.34 & 50 & 25 & 1.10 & 1.08 \\
$\# 5$ & 83.25 & 50 & 25 & 1.08 & 1.0523 \\
$\# 6$ & 84.89 & 50 & 25 & 1.06 & 1.04 \\
\hline 7
\end{tabular}




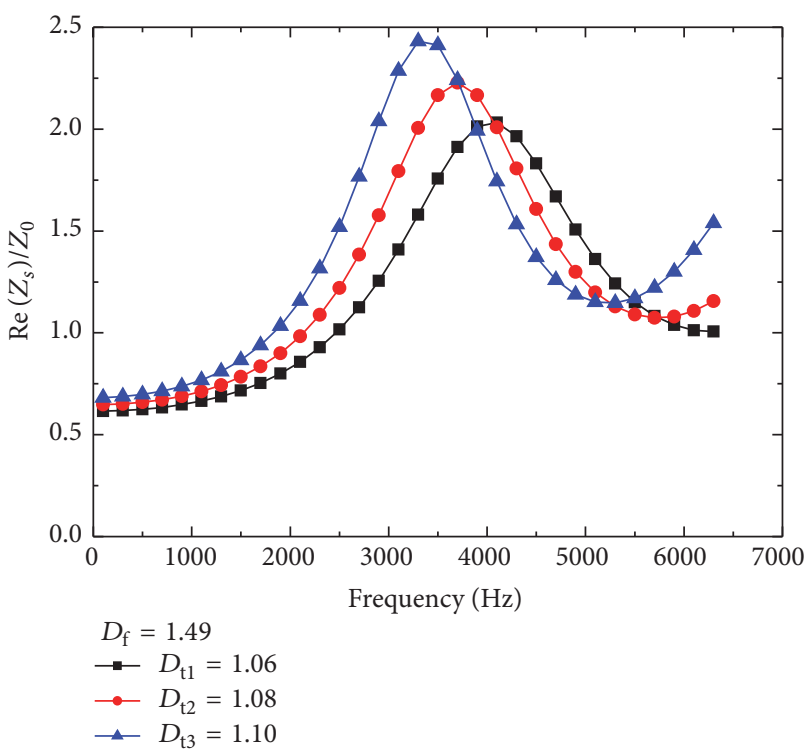

(a)

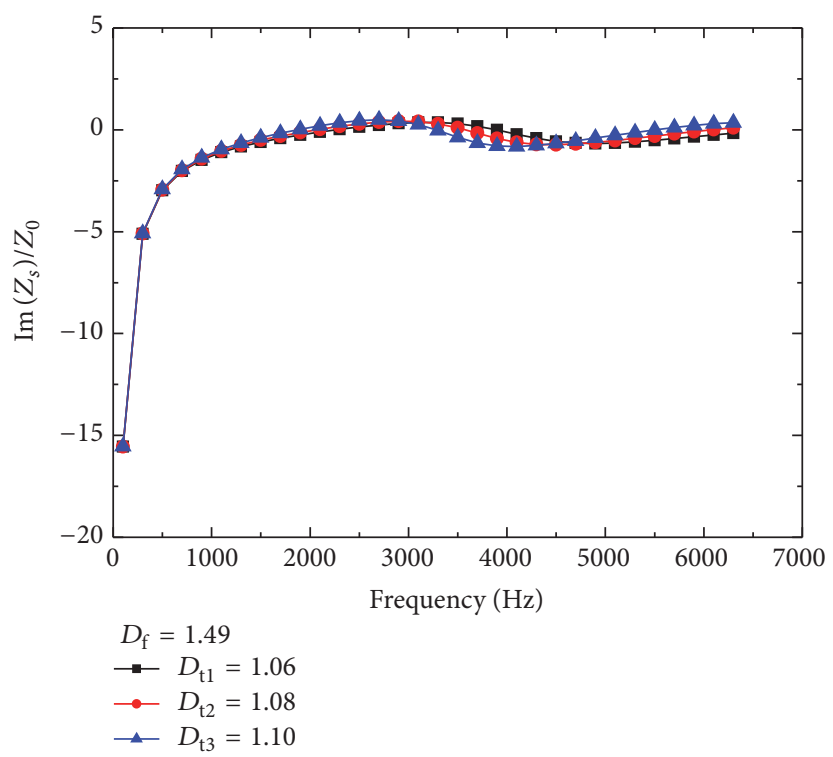

(b)

FIGURE 15: Predictions of specific surface acoustic impedance $Z_{\mathrm{s}} / Z_{0}$ with different tortuosity fractal dimensions. (a) The real part of $Z_{\mathrm{s}} / Z_{0}$. (b) The imaginary part of $Z_{\mathrm{s}} / Z_{0}$.

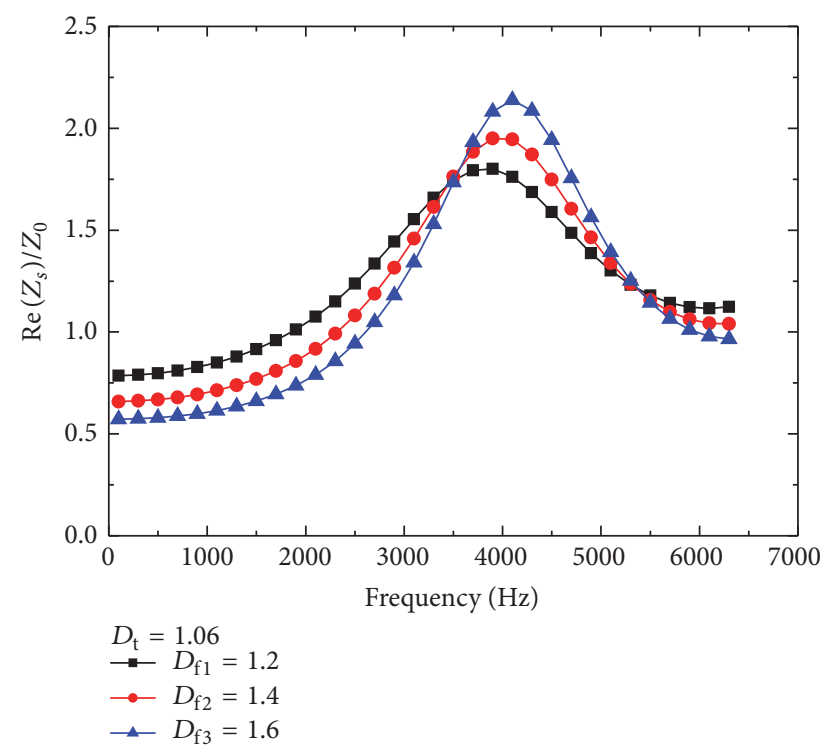

(a)

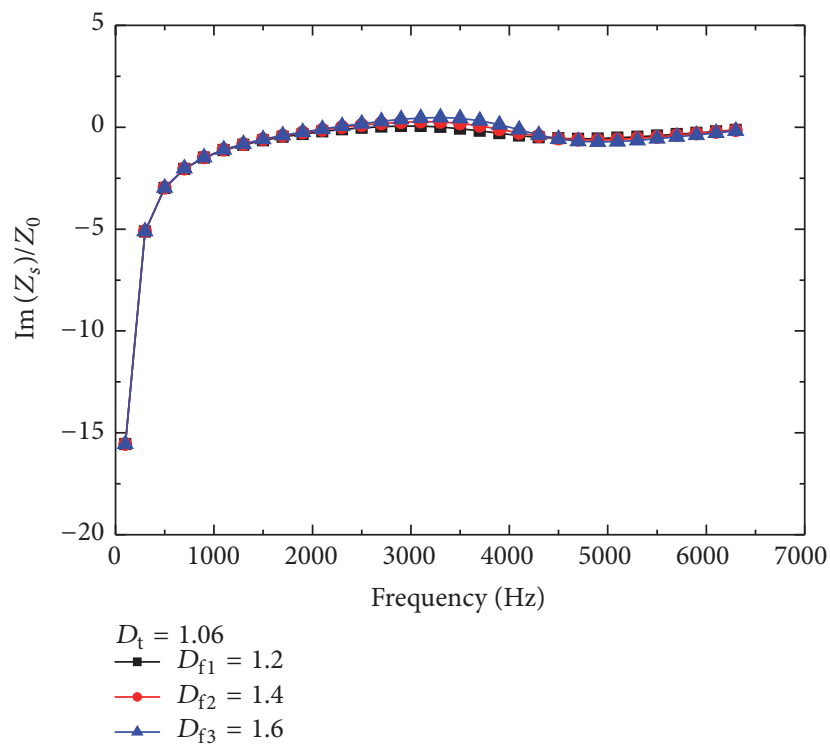

(b)

Figure 16: Predictions of specific surface acoustic impedance $Z_{s} / Z_{0}$ with different pore area fractal dimensions. (a) The real part of $Z_{s} / Z_{0}$. (b) The imaginary part of $Z_{s} / Z_{0}$.

pathways for sound propagation become more tortuous and the static flow resistivity is increased. At $500-2500 \mathrm{~Hz}$ range, the number of sound waves in the PFMM is increased with the tortuosity fractal dimension and more sound energy can be dissipated sufficiently. Therefore, the sound absorption coefficient increases with the tortuosity fractal dimension. When the frequency is increased and reaches the 2500$4800 \mathrm{~Hz}$ range, the wavelength of sound wave is reduced and approaches the porous diameter. Then, it becomes difficult for the sound wave to be incident and the sound absorption coefficient decreases with the tortuosity fractal dimension. Finally, from 4800 to $6400 \mathrm{~Hz}$, the wavelength of sound wave is reduced further and the number of incident sound waves is increased. So the sound absorption coefficient increases again with the tortuosity fractal dimension.

On the other hand, Figure 19(b) plots the changing rules of the sound absorption coefficients with the frequency for different pore area fractal dimensions $\left(D_{\mathrm{f}}=1.2,1.4\right.$, 


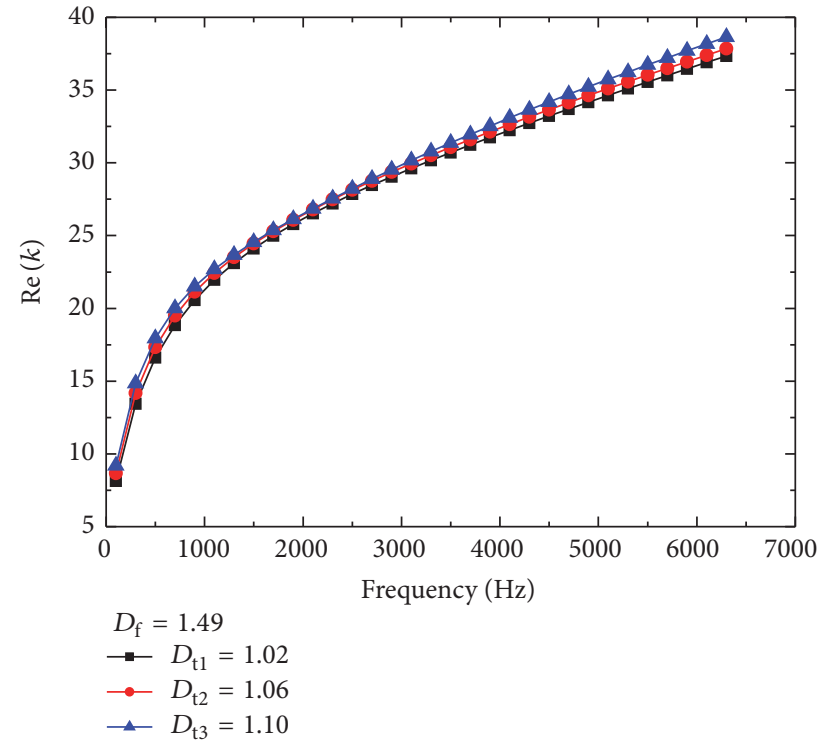

(a)

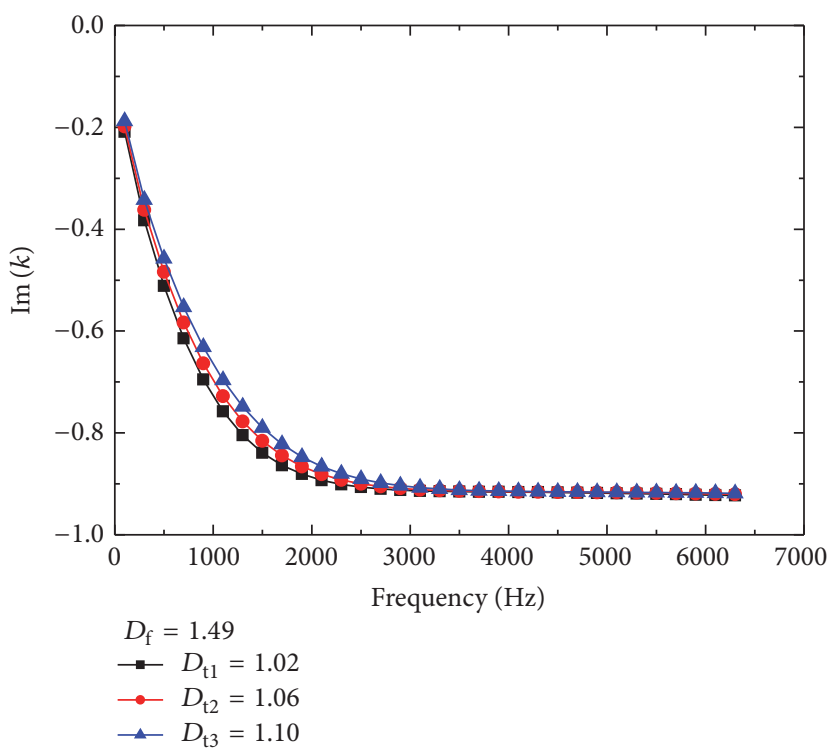

(b)

FIGURE 17: Predictions of the complex wave number $k$ with different tortuosity fractal dimensions. (a) The real part of $k$. (b) The imaginary part of $k$.

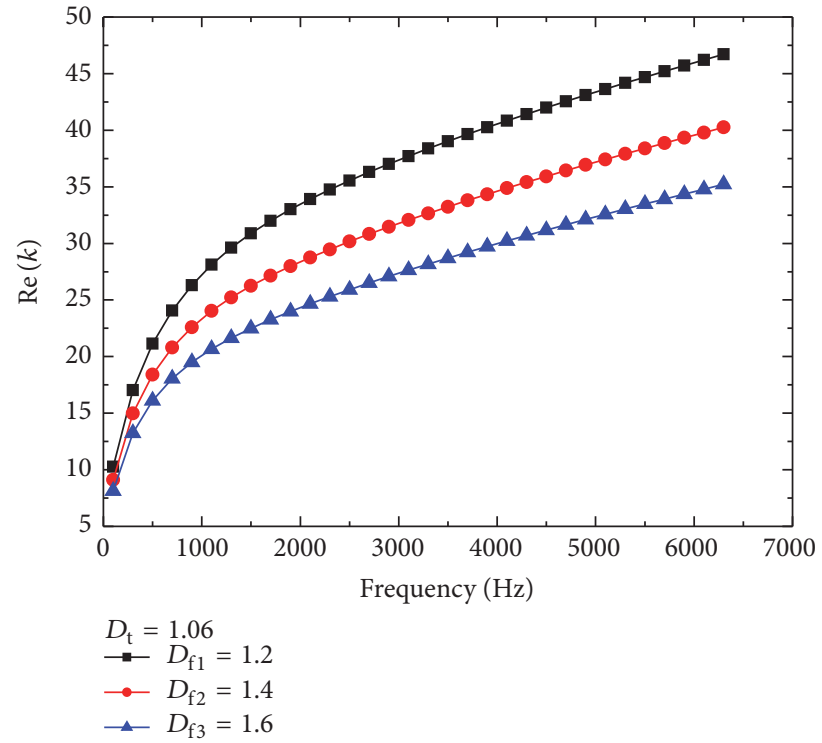

(a)

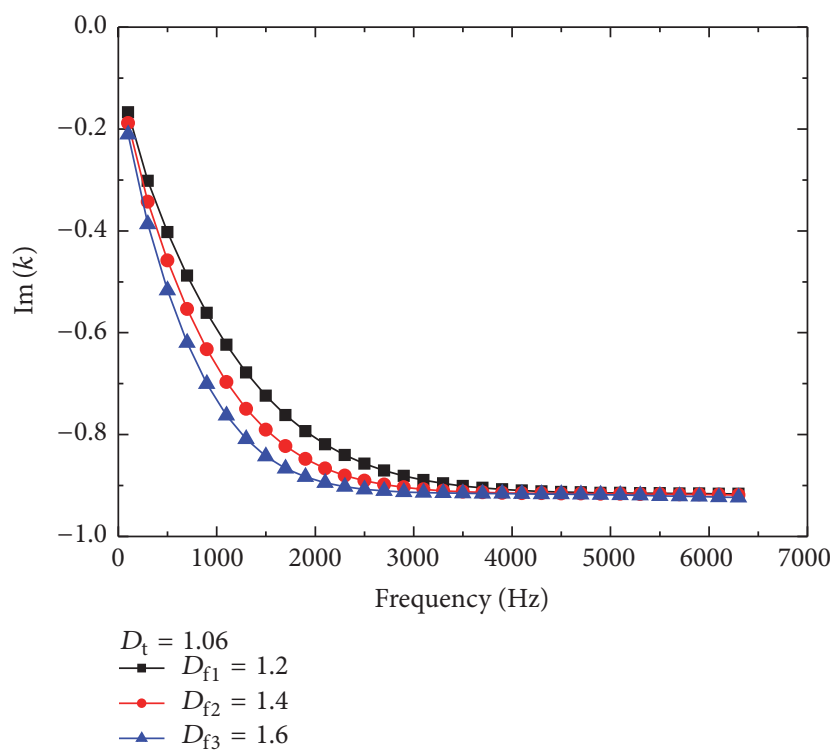

(b)

FIGURE 18: Predictions of the complex wave number $k$ with different pore area fractal dimensions. (a) The real part of $k$. (b) The imaginary part of $k$.

and 1.6) and $D_{t}=1.06$. The results show that the sound absorption coefficient decreases with the pore area fractal dimension in $500-2500 \mathrm{~Hz}$ and $5000-6400 \mathrm{~Hz}$ range but increases in $2500-5000 \mathrm{~Hz}$ range. From 500 to $2500 \mathrm{~Hz}$, the porosity is increased with the pore area fractal dimension and the acoustic resistance plays a major role. Furthermore, the larger the porosity, the smaller the acoustic resistance and the acoustic reactance. Therefore, the sound absorption coefficient decreases with the pore area fractal dimension when the frequency is lower than $2500 \mathrm{~Hz}$. However, when the frequency is higher than $2500 \mathrm{~Hz}$ but lower than $5000 \mathrm{~Hz}$, the acoustic reactance plays a major role. Then, the porosity is increased and the larger the spaces among fibers, the more absorbed the acoustic energy. So the sound absorption coefficient is increased. At $5000-6400 \mathrm{~Hz}$ range, due to the reduction of sound energy attenuation, the sound absorption coefficient decreases again with the increasing of the pore area fractal dimension.

In summary, the sound absorption coefficient is associated with the tortuosity fractal dimension, the pore area 


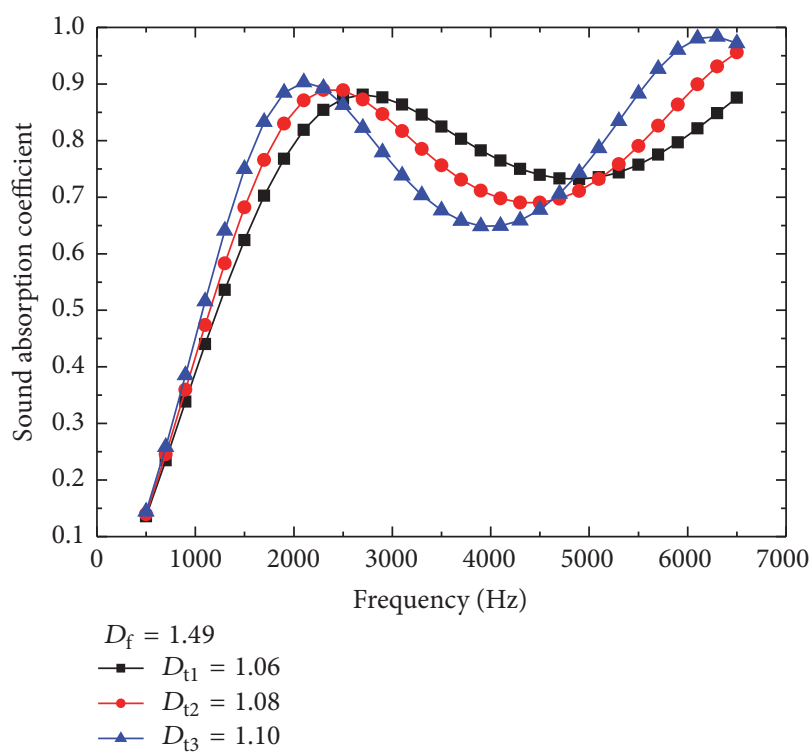

(a)

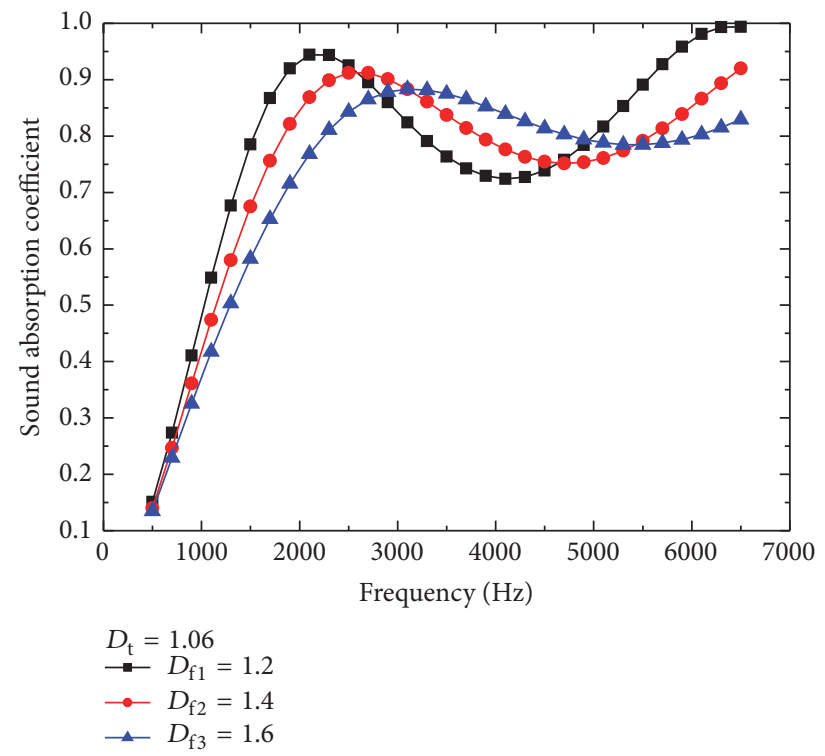

(b)

Figure 19: The Sound absorption coefficient versus the fractal dimensions. (a) $D_{\mathrm{f}}=1.49$ and $D_{\mathrm{t}}=1.06,1.08$, and 1.10, respectively; (b) $D_{\mathrm{t}}=1.06$ and $D_{\mathrm{f}}=1.2,1.4$, and 1.6 , respectively.

fractal dimension, and the porosity. Furthermore, the geometrical parameters such as the pore diameter, the thickness, and the arrangement of fibers are related to the sound absorption performance of the PFMM. Therefore, the optimization of sound absorption capability for the PFMM is dependent on the design of geometrical parameters which are determined by the fractal acoustic model.

\section{Conclusions}

A quantitative fractal acoustic model of the PFMM considering the geometrical microstructure is derived in this paper. The fractal acoustic model is mainly related to the tortuosity fractal dimension and the pore area fractal dimension. Based on the presented acoustic theoretical model, the acoustic performance including sound absorption, the specific surface acoustic impedance, and the complex wave number are investigated both theoretically and experimentally. The experimental results and the values of Biot-Allard model are adopted to validate the theoretical model predictions and a good agreement is achieved. The fractal acoustic theory is also applicable to other materials having a microstructure similar to the PFMMs. The main conclusions are summarized as follows:

(1) In frequency domain, the sound absorption coefficient increases in $100-2000 \mathrm{~Hz}$ and $4000-6400 \mathrm{~Hz}$ range but decreases in $2000-4000 \mathrm{~Hz}$ range. At a fixed value of $D_{\mathrm{f}}$, the sound absorption coefficient increases with the tortuosity fractal dimension in $500-2500 \mathrm{~Hz}$ and $4800-6400 \mathrm{~Hz}$ ranges but decreases from 2500 to $4800 \mathrm{~Hz}$. In addition, the sound absorption coefficient decreases with the pore area fractal dimension in
$500-2500 \mathrm{~Hz}$ and $5000-6400 \mathrm{~Hz}$ ranges but increases from 2500 to $5000 \mathrm{~Hz}$.

(2) The specific acoustic resistance changes obviously with the increase of the tortuosity fractal dimension but the specific acoustic reactance changes slightly in the whole frequency range. The specific acoustic resistance decreases with the pore area fractal dimension in low frequencies but increases in high frequencies; the specific acoustic reactance decreases slightly with the pore area fractal dimension in low frequencies but increases in high frequencies.

(3) Both the real and imaginary parts of the complex wave number increase with the tortuosity fractal dimension. The real part increases with the frequency and decreases with the pore area fractal dimension; the imaginary part decreases with the frequency and the pore area fractal dimension and finally keeps a constant.

\section{Competing Interests}

The authors declare that there are no competing interests regarding the publication of this article.

\section{Acknowledgments}

This work is supported by the National Basic Research Program of China (Grant no. 2011CB610306) and the Natural Science Foundation of Shaanxi Province of China (2015JM5154). The authors are also grateful to the Northwest Institute for Nonferrous Metal Research for providing them with the PFMM samples. 


\section{References}

[1] J. H. Wu, Z. P. Hu, and H. Zhou, "Sound absorbing property of porous metal materials with high temperature and high sound pressure by turbulence analogy," Journal of Applied Physics, vol. 113, no. 19, Article ID 194905, 2013.

[2] F. G. Sun, H. Chen, J. Wu, and K. Feng, "Sound absorbing characteristics of fibrous metal materials at high temperatures," Applied Acoustics, vol. 71, no. 3, pp. 221-235, 2010.

[3] J. P. Arenas and M. J. Crocker, "Recent trends in porous soundabsorbing materials," Sound and Vibration, vol. 44, no. 7, pp. 1217,2010 .

[4] X. L. Wang, F. Peng, and B. J. Chang, "Sound absorption of porous metals at high sound pressure levels," Journal of the Acoustical Society of America, vol. 126, no. 2, pp. EL55-EL61, 2009.

[5] P. Nordin, S. Sarin, and E. Rademaker, "Development of new liner technology for application in hot stream areas of aeroengines," in Proceedings of the 10th AIAA/CEAS Aeroacoustics Conference, AIAA Paper 2004-3033, 2004.

[6] M. A. Biot, "Theory of propagation of elastic waves in a fluidsaturated porous solid. I. Low-frequency range," The Journal of the Acoustical Society of America, vol. 28, pp. 168-178, 1956.

[7] M. A. Biot, "Theory of propagation of elastic waves in a fluidsaturated porous solid II. Higher frequency range," Journal of Acoustical Society of America, vol. 28, pp. 179-191, 1956.

[8] T. Cox and P. D'Antonio, Acoustic Absorbers and Diffusers: Theory, Design and Application, Taylor \& Francis, New York, NY, USA, 2009.

[9] Y. Kawasima, "Sound propagation in a fibre block as a composite medium," Acta Acustica United with Acustica, vol. 10, no. 4, pp. 208-217, 1960.

[10] V. Tarnow, "Compressibility of air in fibrous materials," The Journal of the Acoustical Society of America, vol. 99, no. 5, pp. 3010-3017, 1996.

[11] V. Tarnow, "Calculation of the dynamic air flow resistivity of fiber materials," Journal of the Acoustical Society of America, vol. 102, no. 3, pp. 1680-1688, 1997.

[12] J.-F. Allard and Y. Champoux, "New empirical equations for sound propagation in rigid frame fibrous materials," Journal of the Acoustical Society of America, vol. 91, no. 6, pp. 3346-3353, 1992.

[13] M. E. Delany and E. N. Bazley, "Acoustical properties of fibrous absorbent materials," Applied Acoustics, vol. 3, no. 2, pp. 105-116, 1970.

[14] N. Sebaa, Z. E. A. Fellah, M. Fellah, W. Lauriks, and C. Depollier, "Measuring flow resistivity of porous material via acoustic reflected waves," Journal of Applied Physics, vol. 98, no. 8, Article ID 084901, 2005.

[15] Z. E. A. Fellah, M. Fellah, F. G. Mitri, N. Sebaa, C. Depollier, and W. Lauriks, "Measuring permeability of porous materials at low frequency range via acoustic transmitted waves," Review of Scientific Instruments, vol. 78, no. 11, Article ID 114902, 2007.

[16] M. Sadouki, Z. E. A. Fellah, A. Berbiche et al., "Measuring static viscous permeability of porous absorbing materials," The Journal of the Acoustical Society of America, vol. 135, no. 6, pp. 3163-3171, 2014.

[17] C. Van der Kelen, Characterization of Anisotropic Acoustic Properties of Porous Materials-Inverse Estimation of Static Flow Resistivity, Digitala Vetenskapliga Arkivet, Sweden, Stockholm, 2011.
[18] J.-Z. Wang, Z.-P. Xi, H.-P. Tang, W.-D. Huang, J.-L. Zhu, and Q.B. Ao, "Fractal dimension for porous metal materials of $\mathrm{FeCrAl}$ fiber," Transactions of Nonferrous Metals Society of China, vol. 23, no. 4, pp. 1046-1051, 2013.

[19] J. F. Allard and N. Atalla, Propagation of Sound in Porous Media: Modelling Sound Absorbing Materials, Elsevier Applied Science, London, UK, 1993.

[20] A. J. Katz and A. H. Thompson, "Fractal sandstone pores: implications for conductivity and pore formation," Physical Review Letters, vol. 54, no. 12, pp. 1325-1328, 1985.

[21] B. B. Mandelbrot and W. H. Freeman, The Fractal Geometry of Nature, 1982.

[22] F. M. Borodich and A. B. Mosolov, "Fractal roughness in contact problems," Journal of Applied Mathematics and Mechanics, vol. 56, no. 5, pp. 681-690, 1992.

[23] A. Majumdar and B. Bhushan, "Role of fractal geometry in roughness characterization and contact mechanics of surfaces," Journal of Tribology, vol. 112, no. 2, pp. 205-216, 1990.

[24] R. Pitchumani and B. Ramakrishnan, "A fractal geometry model for evaluating permeabilities of porous preforms used in liquid composite molding," International Journal of Heat and Mass Transfer, vol. 42, no. 12, pp. 2219-2232, 1999.

[25] S. W. Wheatcraft and S. W. Tyler, "An explanation of scaledependent dispersivity in heterogeneous aquifers using concepts of fractal geometry," Water Resources Research, vol. 24, no. 4, pp. 566-578, 1988.

[26] M. M. Denn, Process Fluid Mechanics, Prentice-Hall, Englewood Cliffs, NJ, USA, 1980.

[27] B. M. Yu and P. Cheng, "A fractal permeability model for bidispersed porous media," International Journal of Heat and Mass Transfer, vol. 45, no. 14, pp. 2983-2993, 2002.

[28] T. Ai, R. Zhang, H. W. Zhou, and J. L. Pei, "Box-counting methods to directly estimate the fractal dimension of a rock surface," Applied Surface Science, vol. 314, pp. 610-621, 2014.

[29] ISO, "Acoustics-materials for acoustical applicationsdetermination of airflow resistance," International Standard ISO 9053:1991, International Organization for Standardization, Geneva, Switzerland, 1991.

[30] S. T. Liu, W. J. Chen, and Y. C. Zhang, "Design optimization of porous fibrous material for maximizing absorption of sounds under set frequency bands," Applied Acoustics, vol. 76, pp. 319$328,2014$. 


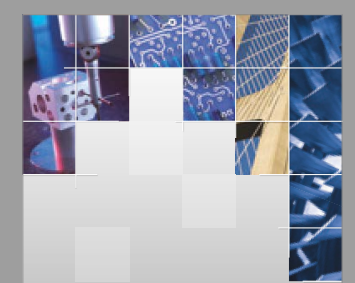

\section{Enfincering}
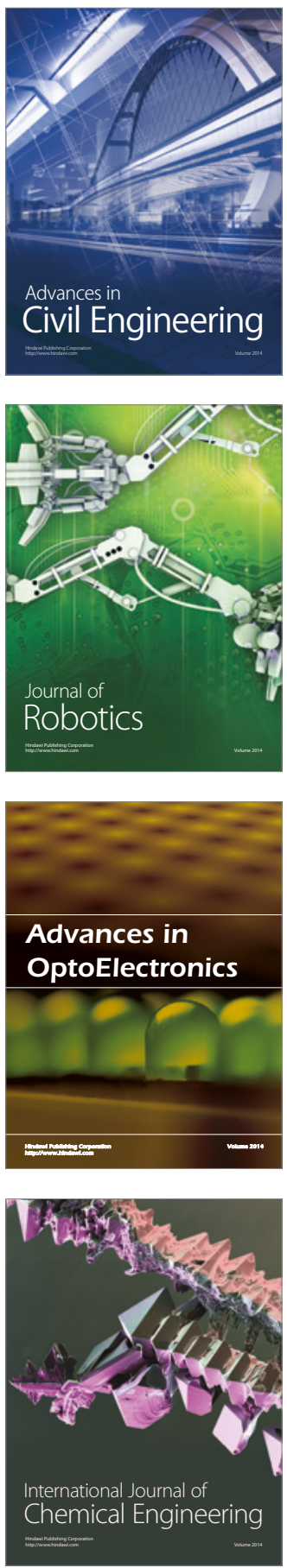

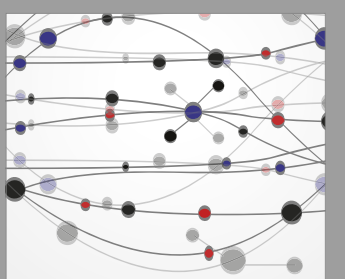

The Scientific World Journal

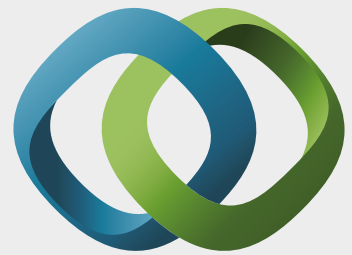

\section{Hindawi}

Submit your manuscripts at

https://www.hindawi.com
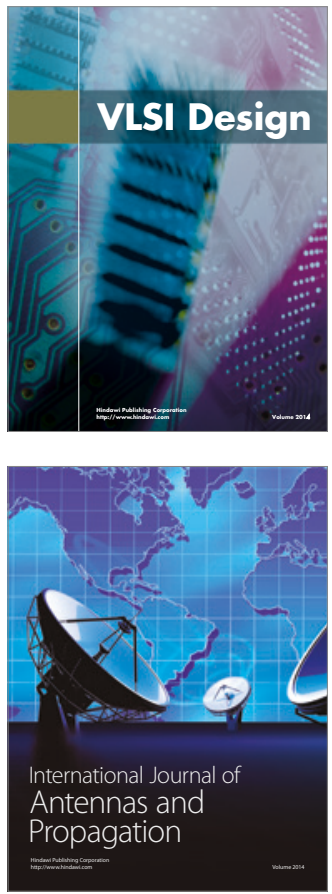

\section{Rotating}

Machinery
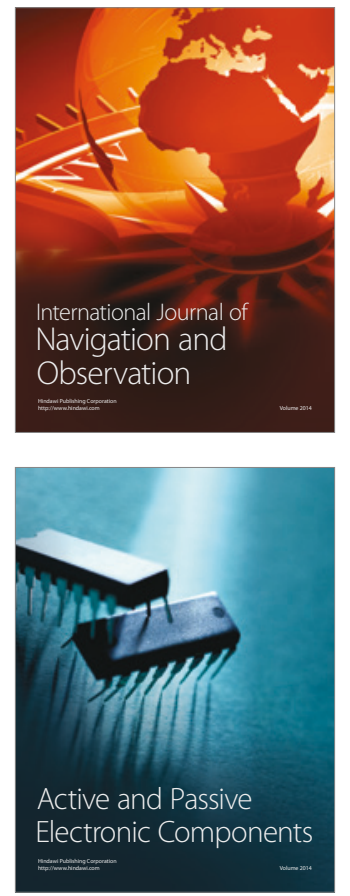
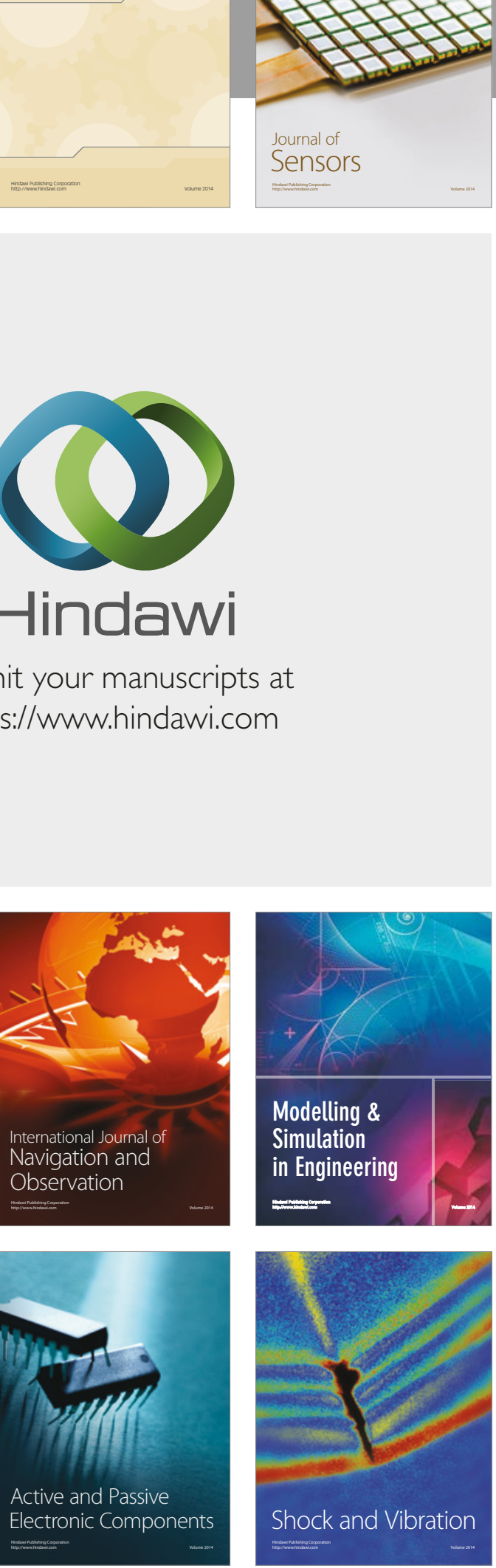
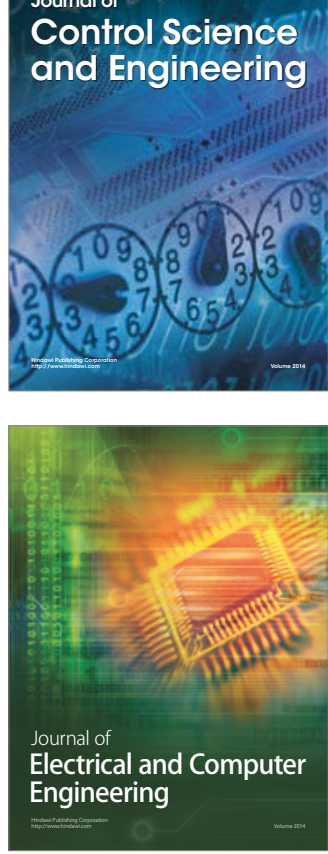

Distributed

Journal of

Control Science

and Engineering
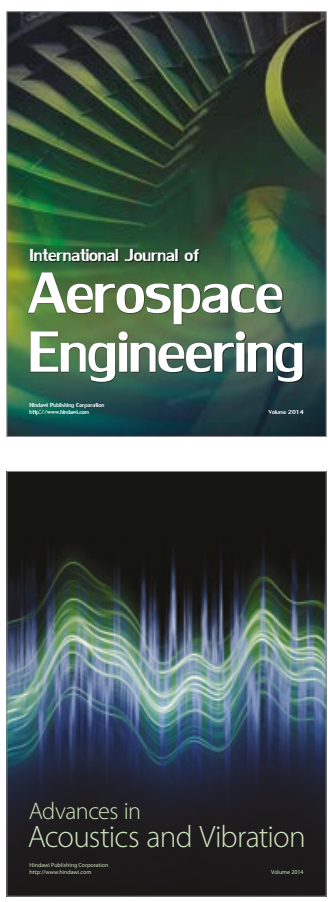

Sensor Networks 